\title{
Intravitreal Therapy for Diabetic Macular Edema: An Update
}

\author{
Claudio Furino $\mathbb{D},{ }^{1}$ Francesco Boscia $\left(\mathbb{D},{ }^{1}\right.$ Michele Reibaldi $\mathbb{D},^{2}$ and Giovanni Alessio $\mathbb{D}^{1}$ \\ ${ }^{1}$ Department of Medical Science, Neuroscience and Sense Organs, Eye Clinic, University of Bari, \\ Azienda Ospedaliero-Universitaria Policlinico Bari, Bari, Italy \\ ${ }^{2}$ Department of Surgical Sciences, Eye Clinic, University of Torino, Torino, Italy
}

Correspondence should be addressed to Claudio Furino; claudiofurino@gmail.com

Received 14 November 2020; Revised 11 February 2021; Accepted 16 February 2021; Published 23 February 2021

Academic Editor: Enrique Mencía-Gutiérrez

Copyright ( $\odot 2021$ Claudio Furino et al. This is an open access article distributed under the Creative Commons Attribution License, which permits unrestricted use, distribution, and reproduction in any medium, provided the original work is properly cited.

\begin{abstract}
Diabetic macular edema (DME) represents a prevalent and disabling eye condition. Despite that DME represents a sightthreatening condition, it is also among the most accessible to treatment. Many different treatment options including photocoagulation, intravitreal medical treatment (either vascular endothelial growth factor inhibitors or corticosteroids therapies), and surgical removal are currently available. Although laser has been considered as the gold standard for many years, over the past several years vascular endothelial growth factor inhibitors (anti-VEGFs) have become first-line therapy. However, many patients do not adequately respond to them. With the development of sustained-release corticosteroid devices, steroids have gained a presence in the management of the DME. We review and update the role of anti-VEGF and intravitreal sustained-release corticosteroid management of DME. According to the currently available scientific evidence, the choice of one anti-VEGF over another critically depends on the baseline best-corrected visual acuity (BCVA). While aflibercept may be the drug of choice in low baseline BCVA, the three anti-VEGFs (bevacizumab, ranibizumab, and aflibercept) provided similar functional outcomes when the baseline BCVA was higher. DEX implants are a valuable option for treating DME, although they are usually seen as a second choice, particularly in those eyes that have an insufficient response to anti-VEGF. The new evidence suggested that, in eyes that did not adequately respond to anti-VEGF, switching to a DEX implant at the time to 3 monthly anti-VEGF injections provided better functional outcomes.
\end{abstract}

\section{Introduction}

Because the prevalence of diabetes is rising, the relevance of diabetic eye disease increases $[1,2]$. In Europe, it was estimated that approximately 6.4 million people are currently affected by any diabetic eye disease and 8.6 million people will be affected in 2050 [3]. In the year 2020, moderate to severe visual impairment due to diabetic retinopathy has been estimated in $4.06 \%$ (Western Europe), 4.77\% (AsiaPacific, high income), and 4.99\% (United States, high income) [2].

Diabetic macular edema (DME) is a chronic, multifactorial, and sight-threatening condition that critically impacts patients' quality of life $[4,5]$.

The prevalence of any DME in Europe was 3.7\% and its pooled mean annual incidence in type 2 diabetes patients was $0.4 \%[3]$.
Despite that DME represents a sight-threatening condition, it is also among the most accessible to treatment. Many different treatment options including laser photocoagulation, intravitreal medical treatment (either vascular endothelial growth factor inhibitors or corticosteroids therapies), and pars plana vitrectomy [6] are currently available.

A retrospective analysis, conducted on a population of 13,410 treatment-naïve DME patients, has found that the treatment patterns within 28 days of initial DME diagnosis were as follows: observation in 9,990 (74.5\%) patients, vascular endothelial growth factor inhibitors (anti-VEGFs) in $2,086(15.6 \%)$ patients, laser in $1,133(8.4 \%)$ patients, corticosteroids in $133(1.0 \%)$ patients, and combined treatment in $68(0.5 \%)$ patients [7].

The main purpose of this paper was to review the role of anti-VEGF and intravitreal sustained-release corticosteroid 
devices for treating patients with DME. Additionally, this paper is also going to evaluate the current evidence about the convenience of switching to intravitreal dexamethasone implant in those patients with suboptimal response to antiVEGF therapies.

\section{Pathophysiology}

A complete review of the pathophysiologic mechanisms in $\mathrm{DME}$ is beyond the scope of this paper.

Macular edema (ME) is defined as an abnormal increase of fluid volume in the macula [8]. The etiology and pathogenesis of DME are multifactorial and result from multiple and intricate mechanisms. Although hyperglycemia is the main risk factor for diabetic retinopathy, different factors including hypoxia, impaired blood flow, retinal ischemia, and inflammation are also associated with DME $[8,9]$.

Different molecules such as interleukin-6 (IL-6), IL-8, IL-1B, vascular endothelial growth factor (VEGF), and tumor necrosis factor- $\alpha$ (TNF- $\alpha$ ) are upregulated in eyes with $\operatorname{DME}[9,10]$. These mechanisms cause a disruption of the blood-retinal barrier that not only leads to the accumulation of subretinal and intraretinal fluid but also stimulates the expression of adhesion molecules that facilitate the adhesion capacity of inflammatory cells $[9,10]$.

Recent investigations have shown that chronic hyperglycemia induces oxidative stress and inflammation in the retina, which constitutes early processes in the development of DME $[8,11]$. Increased inflammation is associated with capillary nonperfusion and breakdown of the blood-retina barrier $[8,10,11]$. Inflammation is not only a consequence of barrier dysfunction but also an early local mechanism contributing to barrier alteration and leukostasis $[8,10,11]$.

Inflammatory cytokines, which mediate vascular permeability, such as tumor necrosis factors alpha and beta, alpha 4 integrin, nitric oxide, and interleukin- $1 \beta$ are elevated in DME [8-11] (Figure 1).

\section{Treatment Strategies of DME}

Although laser treatment has been considered as the gold standard for many years $[12,13]$, according to the European Society of Retina Specialists (EURETINA) guidelines, focal/ grid laser is now reserved mostly for non-center-involving DME [6].

Currently, anti-VEGF agents are considered the first line of treatment in center-involving DME; however, all the large clinical trials have shown that only $33-45 \%$ of DME patients on anti-VEGF agents show 3 lines or more of visual improvement $[6,14,15]$. The inadequate response to antiVEGF observed in many patients speaks in favor of the presence of other factors beyond VEGF, such as inflammation, which is not targeted by the anti-VEGF drugs. There is, therefore, a need for supplemental treatments that might improve visual acuity in eyes with persistent edema despite anti-VEGF therapy.

An overview of the currently available and future options for treating DME is summarized in Figure 2.
3.1. Vascular Endothelial Growth Factor Inhibitors. The introduction of anti-VEGF agents has revolutionized the medical management of DME. Under the umbrella of the term "anti-VEGF," there are several different molecules that can be classified as aptamers (pegaptanib), antibodies to VEGF (bevacizumab), antibody fragments to VEGF (ranibizumab), and fusion proteins, which combine a receptor for VEGF with the constant region of a human immunoglobulin (aflibercept and conbercept) [14, 15].

3.1.1. Pegaptanib. Pegaptanib (Macugen ${ }^{\circledR}$, Bausch and Lomb, Rochester, NY, USA), developed to bind and block the activity of extracellular VEGF $[16,17]$, was the first commercially available anti-VEGF drug used to treat DME [17]. Cunningham et al. [17] in a randomized, doublemasked, multicenter, dose-ranging, and sham-controlled phase II trial evaluated the efficacy and safety of pegaptanib in the treatment of DME. Three different doses of pegaptanib $(0.3 \mathrm{mg}, 1.0 \mathrm{mg}$, and $3.0 \mathrm{mg})$ were tested and compared to sham injections. Injections were planned at baseline, week 6, and week 12, with additional injections and/or focal photocoagulation as needed for another 18 weeks [17]. At week 36 compared with baseline, visual acuity improvement $\geq 10$ letters occurred in 15 of $44(34 \%)$ patients, 13 of $43(30 \%)$ patients, 6 of $42(14 \%)$ patients, and 4 of 41 (10\%) patients in the $0.3 \mathrm{mg}, 1.0 \mathrm{mg}, 3.0 \mathrm{mg}$, and sham subgroups, respectively ( $p=0.003,0.3 \mathrm{mg}$ versus sham) [17].

Sultan et al. [18] evaluated in a randomized $(1: 1)$, shamcontrolled, multicenter, parallel-group clinical trial the efficacy and safety of intravitreal pegaptanib $0.3 \mathrm{mg}$ versus sham injections. Based on the results of this study, the probability of achieving a visual acuity improvement of $\geq 10$ letters, at week 54 compared with baseline, was significantly greater in the pegaptanib group (odds ratio, 2.38; 95\% confidence interval, 1.32-4.30; $p=0.0047$ ) [18].

Additionally, the results of a Japanese phase III randomized clinical trial found that the proportion of patients who achieved a visual acuity improvement of $\geq 10$ letters, from baseline to week 24, was significantly greater in the pegaptanib group $(20.3 \%)$ than in the sham group $(5 \%)$, $p=0.0003$ ) [19].

The results of these randomized clinical trials have been confirmed in several studies conducted in clinical settings [20-22].

Similarly, the results of a retrospective study, which evaluated the effect of intravitreal pegaptanib on the functional and anatomical outcomes, found a significant improvement (from baseline to the last follow-up visit) in mean BCVA and a significant reduction in central macular thickness (CMT) $(p<0.001$ and $p<0.001$, respectively) [20].

Rinaldi et al. [21] published in 2012 a longitudinal, interventional, and nonrandomized study that evaluated the efficacy and safety of intravitreal pegaptanib in patients with "clinically significant diabetic macular edema". The results of this study found a significant reduction in foveal thickness $(p=0.0001)$ and a significant improvement in BCVA $(p<0.005)$, macular sensitivity $(p<0.001)$, and color discrimination $(p=0.0001)$ [21]. 


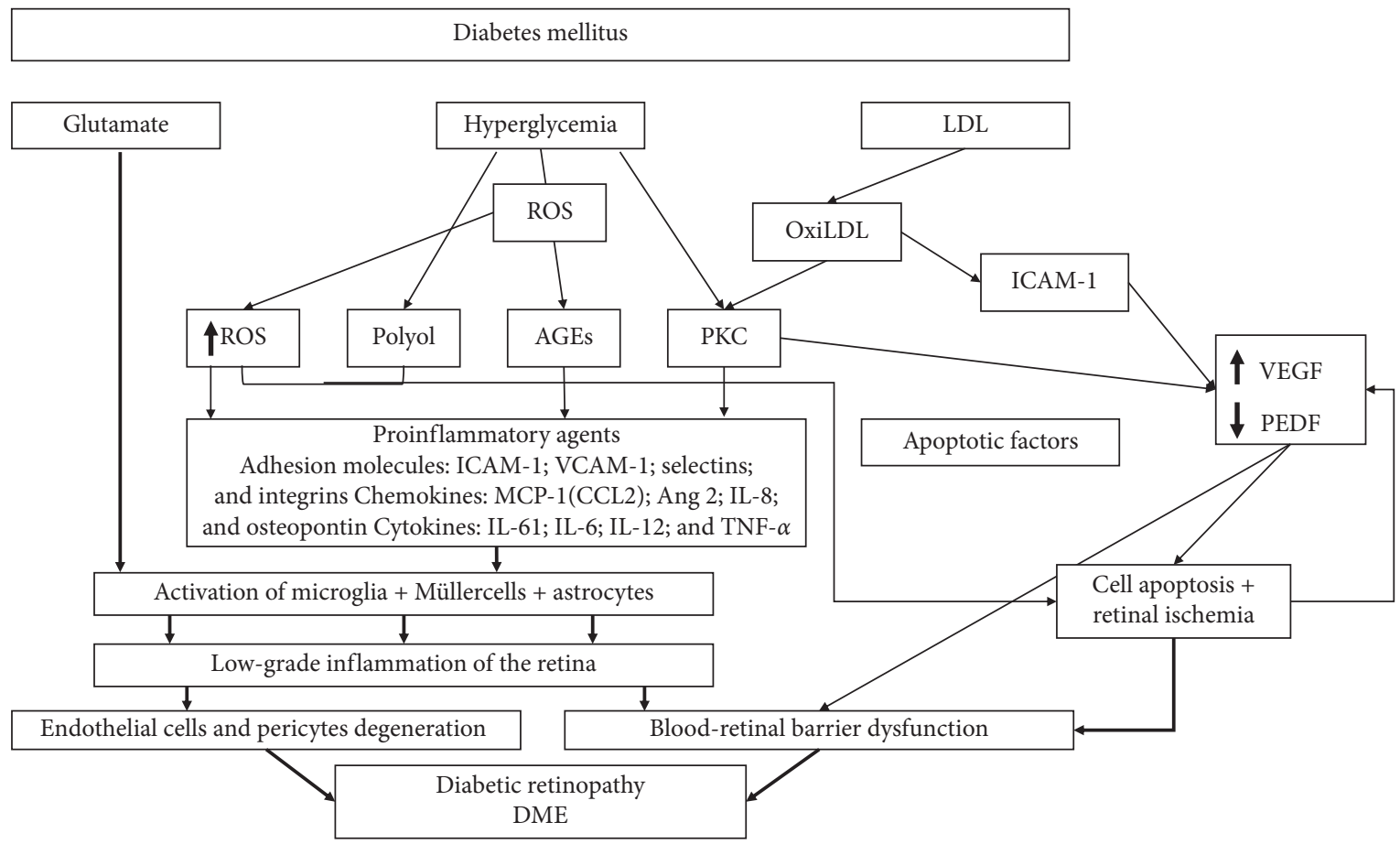

FIGURE 1: An overview of the different pathways involved in the development of diabetic macular edema (adapted from Daruich et al. [8] and Romero-Aroca et al. [9]). LDL: low-density lipoprotein; ROS: reactive oxidative species; Oxi: oxidized; AGEs: advanced glycation endproducts; PKC: protein kinase C; ICAM-1: inflammatory intercellular adhesion molecule-1; VEGF: vascular endothelial growth factor; VCAM-1: vascular cell adhesion molecule-1; PEDF: pigment epithelium-derived factor; CCL2: chemokine C-C motif ligand 2; Ang-2: angiopoietin-2; IL: interleukin; TNF: tumor necrosis factor; DME: diabetic macular edema.

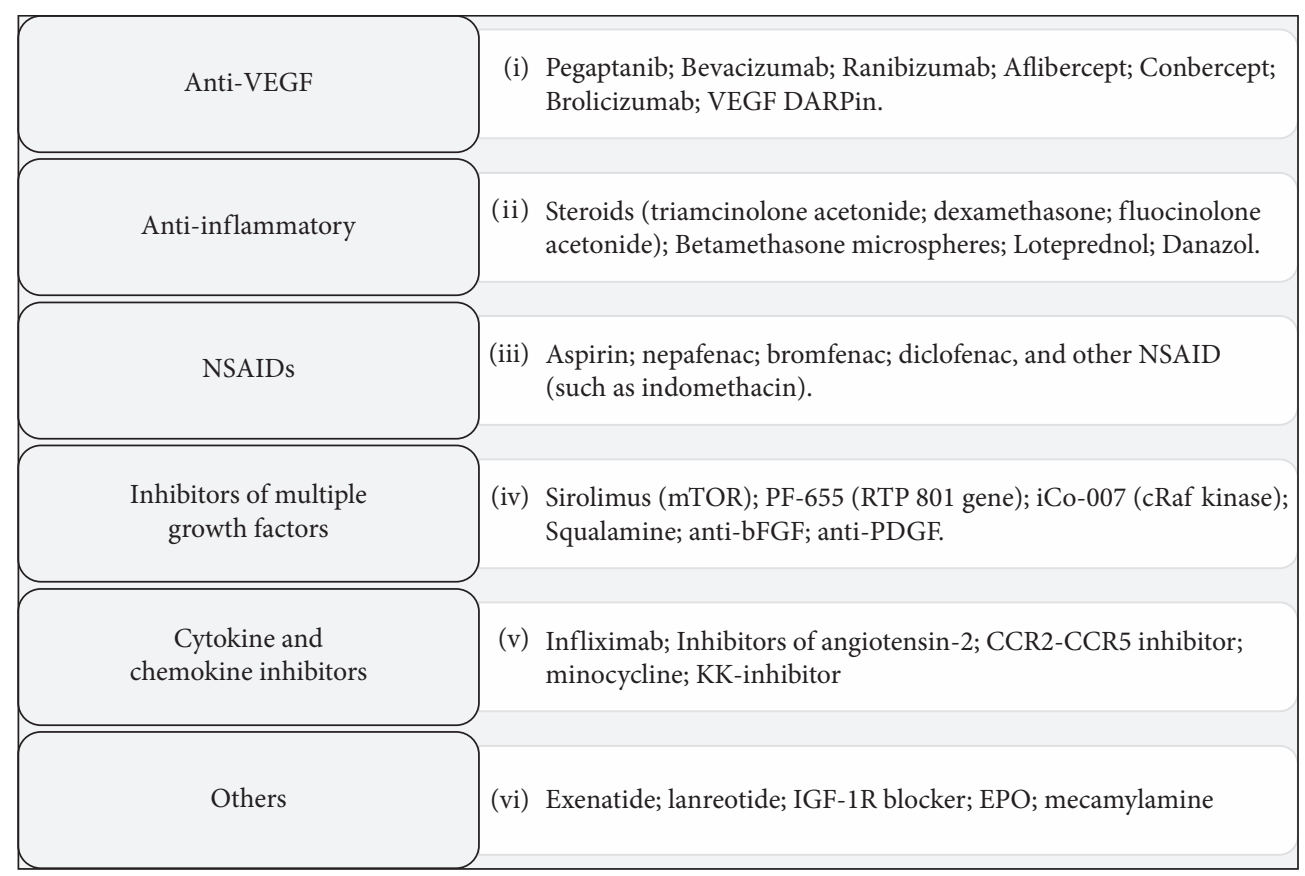

FIGURE 2: Overview of the medical treatment options for diabetic macular edema (adapted from Urias et al. [15]). NSAIDS: nonsteroidal anti-inflammatory drug; VEGF: vascular endothelial growth factor; DARPin: designed ankyrin repeat protein; FGF: fibroblast growth factor beta; PDGF: platelet-derived growth factor; CCR: chemokine receptor; IGF-1: insulin-like growth factor-1; EPO: erythropoietin.

Sivaprasad et al. [22], in an open-label, one-year, and noncomparative study, evaluated the safety and tolerability of pegaptanib in DME patients. Four (25\%) of 12 patients achieved a visual acuity improvement of $\geq 10$ letters and 8 $(8.7 \%)$ of 46 patients reported treatment-related adverse events [22]. 
3.1.2. Bevacizumab. Bevacizumab, a humanized monoclonal antibody that inhibits vascular endothelial growth factor (VEGF), was originally developed as a concomitant medication for use in combination with existing metastatic colorectal cancer regimens [23].

Intravitreal injections of bevacizumab have been and currently continue to be widely used as an off-label treatment for neovascular age-related macular degeneration and DME [6, 7, 14, 15].

The first study evaluating the efficacy of bevacizumab (Avastin; Genentech, Inc., South San Francisco, CA) for the treatment of persistent DME was published by Haritoglou et al. [24]. The results of this prospective, consecutive, and noncomparative case series study found a significant improvement in visual acuity $(p=0.001)$ and a significant reduction in CMT $(p=0.002)$ [24].

These findings were confirmed by different small studies [25-28].

The Diabetic Retinopathy Clinical Research Network (DRCR.net) published in 2007 the results of a randomized phase II clinical trial that evaluated the efficacy and safety of intravitreal bevacizumab (either alone or in combination with focal photocoagulation) in DME patients [29]. The results of this study suggested some positive findings associated with the use of bevacizumab. However, this response was similar to that observed in the laser group after more than 3 weeks [29].

The BOLT (bevacizumab or laser therapy) study was a prospective, randomized, masked, single-center, 2-year, and 2 -arm clinical trial that compared the effect of repeated intravitreal injections of bevacizumab versus (vs.) modified Early Treatment of Diabetic Retinopathy Study (ETDRS) macular laser therapy in patients with persistent clinically significant DME [30]. The results of this study found that, after 12 months of follow-up, the probability of achieving a visual acuity improvement of $\geq 10$ ETDRS letters was significantly greater in the bevacizumab group than in the laser group (adjusted odds ratio, 5.1; 95\% confidence interval, 1.3 to $19.7, p=0.019)$ [30].

The 2-year outcomes of the BOLT study confirmed the aforementioned 12-month data [30]. At 2 years, the proportion of patients gained $\geq 10$ or $\geq 15$ ETDRS letters was significantly greater in the bevacizumab group than in the laser one ( $p=0.001$ and $p=0.004$, respectively) [31].

Different randomized clinical trials (RCTs), whose results have been summarized in Table 1, have evaluated the efficacy and safety of intravitreal bevacizumab in DME patients [29-34]. Although, on average, intravitreal bevacizumab has shown a positive impact on DME patients, in many studies it was not superior to other therapies.

The Protocol $\mathrm{T}$ was a prospective, randomized, and multicenter clinical trial that compared the efficacy and safety of intravitreal injections of bevacizumab, ranibizumab, and aflibercept for the treatment of DME [35]. Patients included in Protocol T were randomly assigned in a $1: 1: 1$ ratio to be injected with bevacizumab $(1.25 \mathrm{mg})$, ranibizumab $(0.3 \mathrm{mg})$, or aflibercept $(2.0 \mathrm{mg})$ [35]. The mean visual acuity improvement was significantly greater with aflibercept than with bevacizumab $(p<0.001)$ and ranibizumab $(p=0.03)$. However, this advantage was not considered as clinically relevant because the effect of visual acuity varied according to the baseline visual acuity [35]. As regards central subfield thickness, aflibercept achieved the greatest reduction with $169 \pm 138 \mu \mathrm{m}(p<0.001$ vs. bevacizumab and $p=0.0336$ vs. ranibizumab), followed by ranibizumab with $147 \pm 134 \mu \mathrm{m}(p<0.001$ vs. bevacizumab $)$ and bevacizumab with $101 \pm 121 \mu \mathrm{m}$. However, similarly to visual acuity, the effect on central subfield thickness varied according to initial visual acuity [35].

The main findings of the Protocol T are shown in Table 2.

The 2-year Protocol $T$ results showed slight changes as compared to the 1-year results. However, as shown in the 1year results, BCVA improvement varied according to the initial visual acuity [36].

(1) Safety. Many ophthalmologists have been and currently are worried about the safety profile of bevacizumab. When used to treat certain cancers, intravenous bevacizumab has been associated with several side effects, including systemic hypertension, proteinuria, and cardiovascular and gastrointestinal complications [37].

The results of a post hoc analysis of the Protocol T found that aflibercept and bevacizumab induced greater decreases in plasma free-VEGF than ranibizumab at 4 weeks [38]. Moreover, at 52 and 104 weeks, a greater decrease was observed in bevacizumab versus ranibizumab [38]. Interestingly, this study did not find any significant relationship between VEGF concentration and the incidence of a heart attack or stroke [28].

The incidence of serious ocular and nonocular adverse events was approximately below 1 per 100 injections for intravitreal bevacizumab [28-36]. Several systemic adverse events have been reported in different studies, including systemic hypertension, cerebrovascular accidents, heart attacks, and death [28-36, 39-41]. Ocular side effects included bacterial endophthalmitis, ocular inflammation (iritis, iridocyclitis, uveitis, or vitreous), tractional retinal detachment, pigmentary epithelial detachment, vitreous hemorrhage, ocular hypertension, and cataract [28-36, 39-41].

According to the European Society of Retina Specialists (EURETINA) guidelines, there are still some issues about the anti-VEGF safety profile of bevacizumab that should be further explored [6].

3.1.3. Ranibizumab. Ranibizumab (Lucentis ${ }^{\circledR}$, Novartis, Basel, Switzerland) is a fully humanized monoclonal antibody fragment, which binds to multiple variants of VEGF-A [42]. It was originally approved for treating neovascular agerelated macular degeneration [6].

The first prospective, randomized, interventional, and multicenter clinical trial comparing the effect of ranibizumab with focal/grid laser or a combination of both in DME was the Ranibizumab for Edema of the mAcula in diabetes (READ-2) study [43]. Patients were randomly assigned in a $1: 1: 1$ regime to receive ranibizumab $0.5 \mathrm{mg}$ (baseline and months 1, 3, and 5), laser (baseline and month 3, if needed), 
TABLE 1: Overview of the functional and anatomic results of bevacizumab.

\begin{tabular}{|c|c|c|c|c|c|c|c|c|}
\hline \multirow{2}{*}{ Study } & \multirow{2}{*}{ Ref. } & \multirow{2}{*}{$\begin{array}{l}\text { Duration } \\
\quad(\mathrm{w})\end{array}$} & \multirow{2}{*}{ Regimen } & \multirow{2}{*}{$\begin{array}{c}N \\
\text { (eyes) }\end{array}$} & \multicolumn{2}{|c|}{ BCVA (ETDRS letters) } & \multicolumn{2}{|c|}{ CRT $(\mu \mathrm{m})$} \\
\hline & & & & & Baseline & Change $^{\mathrm{a}}$ & Baseline & Change \\
\hline \multirow{5}{*}{ DRCR.net $^{\mathrm{a}}$} & \multirow{5}{*}{ [29] } & \multirow{5}{*}{12} & Laser & 19 & $64(50$ to 70$)$ & $-1(-6$ to 5$)$ & 441 (354 to 512$)$ & $-40(-146$ to 85$)$ \\
\hline & & & Beva I & 22 & $65(60$ to 70$)$ & $5(1 \text { to } 12)^{*}$ & 397 (320 to 358$)$ & $-56(-120$ to -6$)$ \\
\hline & & & Beva II & 24 & $63(57$ to 71$)$ & $7(4 \text { to } 11)^{*}$ & $446(342$ to 543$)$ & $-47(-125$ to -16$)$ \\
\hline & & & Beva III & 22 & $64(52$ to 68$)$ & $4(-3$ to 7$)$ & 406 (353 to 520$)$ & $-5(-41$ to 53$)$ \\
\hline & & & Beva IV & 22 & $66(57$ to 72$)$ & $0(-5$ to 8$)$ & 389 (308 to 452$)$ & $-40(-103$ to 33$)$ \\
\hline \multirow{4}{*}{ BOLT $^{\mathrm{b}}$} & \multirow{2}{*}[30]{} & \multirow[t]{2}{*}{52} & $\begin{array}{c}\text { Beva } \\
1.25 \mathrm{mg}\end{array}$ & 42 & $55.7(9.7)$ & $8(1 \text { to } 10)^{* *}$ & 507 (145) & $-130(122)$ \\
\hline & & & Laser & 38 & $54.6(8.6)$ & $-0.5(-15$ to 5$)$ & $481(121)$ & $-68(171)$ \\
\hline & \multirow{2}{*}[31]{} & \multirow{2}{*}{104} & $\begin{array}{c}\text { Beva } \\
1.25 \mathrm{mg}\end{array}$ & 42 & $55.7(9.7)$ & $8.6^{* *}$ & 507 (145) & -146 \\
\hline & & & Laser & 38 & $54.6(8.6)$ & 0.5 & $481(121)$ & -118 \\
\hline \multirow{2}{*}{$\begin{array}{l}\text { Nepomuceno } \\
\text { et al. }{ }^{c}\end{array}$} & \multirow{2}{*}[32]{} & \multirow{2}{*}{48} & Beva $1.5 \mathrm{mg}$ & 32 & $0.60(0.05)$ & $0.36(0.05)$ & $451.7(22.3)$ & $-122.0(20.9)$ \\
\hline & & & Rani $0.5 \mathrm{mg}$ & 28 & $0.63(0.06)$ & $0.34(0.04)$ & $421.9(23.1)$ & $-141.0(18.6)$ \\
\hline \multirow{2}{*}{$\begin{array}{l}\text { Kriechbaum } \\
\text { et al. } .^{\mathrm{c}}\end{array}$} & \multirow{2}{*}[33]{} & \multirow{2}{*}{52} & Beva $2.5 \mathrm{mg}$ & 15 & $0.30(0.19$ to 0.42$)$ & $0.18(0.06$ to 0.3$)$ & 505 (438 to 572$)$ & 351 (258 to 445$)$ \\
\hline & & & Triam $8 \mathrm{mg}$ & 15 & $0.32(0.2$ to 0.43$)$ & $0.36(0.19$ to 0.52$)$ & $490(433$ to 547$)$ & $296(224$ to 368$)$ \\
\hline \multirow[t]{2}{*}{ Sonoda et al. ${ }^{\mathrm{c}}$} & \multirow[t]{2}{*}[34]{} & \multirow[t]{2}{*}{12} & $\begin{array}{c}\text { Beva } \\
1.25 \mathrm{mg}\end{array}$ & 26 & $0.48(0.32)$ & $0.40(0.25)$ & $495.7(195.3)$ & $449.7(212.2)$ \\
\hline & & & Triam 4 mg & 25 & $0.40(0.25)$ & $0.31(0.23)$ & $503.9(171.4)$ & $389.4(209.4)$ \\
\hline
\end{tabular}

Note. ${ }^{\mathrm{a}}$ Data are expressed in median (interquartile range). ${ }^{\mathrm{b}}$ Data are expressed in mean (standard deviation). ${ }^{\mathrm{c}}$ Mean (standard deviation) best-corrected visual acuity $(\log M A R)$ at baseline and at the last follow-up visit. ${ }^{*} p<0.01$ vs. laser. ${ }^{* *} p<0.001$ vs. laser. w: weeks; BCVA: best-corrected visual acuity; EDTRS: Early Treatment of Diabetic Retinopathy Study; CRT: central retina thickness; DRCR.net: Diabetic Retinopathy Clinical Research Network; Beva I: intravitreal injection of $1.25 \mathrm{mg}$ of bevacizumab at baseline and 6 weeks; Beva II: intravitreal injection of $2.5 \mathrm{mg}$ of bevacizumab at baseline and 6 weeks; Beva III: intravitreal injection of $1.25 \mathrm{mg}$ of bevacizumab at baseline and sham injection at 6 weeks; Beva IV: intravitreal injection of $1.25 \mathrm{mg}$ of bevacizumab at baseline and 6 weeks with photocoagulation at 3 weeks; Beva: bevacizumab; Rani: ranibizumab; Triam: triamcinolone acetonide.

TABLe 2: Overview of the visual acuity outcomes of the Protocol T.

\begin{tabular}{|c|c|c|c|c|c|c|c|c|c|}
\hline \multirow{2}{*}{$\begin{array}{l}\text { Visual acuity letter } \\
\text { score and Snellen } \\
\text { equivalent }\end{array}$} & \multirow{2}{*}{ Aflibercept } & \multirow{2}{*}{ Bevacizumab } & \multirow{2}{*}{ Ranibizumab } & \multicolumn{2}{|c|}{$\begin{array}{l}\text { Aflibercept vs. } \\
\text { bevacizumab }\end{array}$} & \multicolumn{2}{|c|}{$\begin{array}{l}\text { Aflibercept vs. } \\
\text { ranibizumab }\end{array}$} & \multicolumn{2}{|c|}{$\begin{array}{l}\text { Ranibizumab vs. } \\
\text { bevacizumab }\end{array}$} \\
\hline & & & & $\begin{array}{c}\text { Difference }(95 \% \\
\text { CI) }\end{array}$ & $\begin{array}{c}p \\
\text { value }^{\mathrm{a}}\end{array}$ & $\begin{array}{c}\text { Difference } \\
(95 \% \mathrm{CI})\end{array}$ & $\begin{array}{c}p \\
\text { value }^{\mathrm{a}}\end{array}$ & $\begin{array}{c}\text { Difference } \\
(95 \% \mathrm{CI})\end{array}$ & $\begin{array}{c}p \\
\text { value }^{\mathrm{a}}\end{array}$ \\
\hline \multicolumn{10}{|c|}{ Letter score of $<69$, equivalent to $20 / 50$ or worse, at baseline } \\
\hline Number of eyes & 102 & 102 & 101 & & & & & & \\
\hline \multicolumn{10}{|c|}{ Visual acuity at baseline } \\
\hline $\begin{array}{l}\text { Mean (SD) } \\
\text { letter score } \\
\text { Approximate }\end{array}$ & $56.2(11.1)$ & $56.6(10.6)$ & $56.5(9.9)$ & & & & & & \\
\hline $\begin{array}{l}\text { Snellen } \\
\text { equivalent }\end{array}$ & $20 / 80$ & $20 / 80$ & $20 / 80$ & & & & & & \\
\hline \multicolumn{10}{|c|}{ Visual acuity at 1 year } \\
\hline $\begin{array}{l}\text { Mean (SD) } \\
\text { letter score }\end{array}$ & $75.2(10.9)$ & $68.5(13.6)$ & $70.7(12.0)$ & & & & & & \\
\hline Approximate & & & & & & & & & \\
\hline $\begin{array}{l}\text { Snellen } \\
\text { equivalent }\end{array}$ & $20 / 32$ & $20 / 40$ & $20 / 40$ & & & & & & \\
\hline \multicolumn{10}{|c|}{ Change from baseline in letter score } \\
\hline $\begin{array}{l}\text { Mean (SD) } \\
\text { improvement }\end{array}$ & $18.9(11.5)$ & $11.8(12.0)$ & $14.2(10.6)$ & $6.5(2.9$ to 10.1$)$ & $<0.001$ & $4.7(1.4$ to 8.0$)$ & 0.003 & $\begin{array}{c}1.8 \\
(-1.1 \text { to } 4.8)\end{array}$ & 0.21 \\
\hline $\begin{array}{l}\text { Improvement } \\
\text { of } \geq 10 \text { letters, } \\
n(\%)\end{array}$ & $79(77)$ & $61(60)$ & $70(69)$ & $17(2$ to 31$)$ & 0.02 & $10(-4$ to 23$)$ & 0.20 & $7(-6$ to 20$)$ & 0.28 \\
\hline $\begin{array}{l}\text { Worsening of } \\
\geq 10 \text { letters, } n \\
(\%)\end{array}$ & $1(1)$ & $4(4)$ & $2(2)$ & $-3(-7$ to 2$)$ & 0.56 & $-1(-5$ to 3$)$ & 0.56 & $-1(-6$ to 3$)$ & 0.56 \\
\hline $\begin{array}{l}\text { Improvement } \\
\text { of } \geq 15 \text { letters, } \\
n(\%)\end{array}$ & $68(67)$ & $42(41)$ & $50(50)$ & 24 (9 to 39$)$ & $<0.001$ & 18 (4 to 32$)$ & 0.008 & $6(-7$ to 19$)$ & 0.34 \\
\hline
\end{tabular}


TABLE 2: Continued.

\begin{tabular}{|c|c|c|c|c|c|c|c|c|c|}
\hline \multirow{2}{*}{$\begin{array}{l}\text { Visual acuity letter } \\
\text { score and Snellen } \\
\text { equivalent }\end{array}$} & \multirow{2}{*}{ Aflibercept } & \multirow{2}{*}{ Bevacizumab } & \multirow{2}{*}{ Ranibizumab } & \multicolumn{2}{|c|}{$\begin{array}{l}\text { Aflibercept vs. } \\
\text { bevacizumab }\end{array}$} & \multicolumn{2}{|c|}{$\begin{array}{l}\text { Aflibercept vs. } \\
\text { ranibizumab }\end{array}$} & \multicolumn{2}{|c|}{$\begin{array}{l}\text { Ranibizumab vs. } \\
\text { bevacizumab }\end{array}$} \\
\hline & & & & $\begin{array}{c}\text { Difference }(95 \% \\
\text { CI) }\end{array}$ & $\underset{\text { value }^{\mathrm{a}}}{p}$ & $\begin{array}{l}\text { Difference } \\
(95 \% \text { CI })\end{array}$ & $\underset{\text { value }^{\mathrm{a}}}{p}$ & $\begin{array}{l}\text { Difference } \\
(95 \% \text { CI })\end{array}$ & $\underset{\text { value }^{\mathrm{a}}}{p}$ \\
\hline $\begin{array}{l}\text { Worsening of } \\
\geq 15 \text { letters, } n \\
(\%)\end{array}$ & $1(1)$ & $2(2)$ & $2(2)$ & $0(-3$ to 3$)$ & 0.85 & $-1(-4$ to 2$)$ & 0.85 & $1(-3$ to 4$)$ & 0.85 \\
\hline \multicolumn{10}{|c|}{ Letter score of 78 to 69 , equivalent to $20 / 32$ to $20 / 40$, at baseline } \\
\hline Number of eyes & 106 & 104 & 105 & & & & & & \\
\hline \multicolumn{10}{|c|}{ Visual acuity at baseline } \\
\hline $\begin{array}{l}\text { Mean (SD) } \\
\text { letter score } \\
\text { Approximate }\end{array}$ & $73.5(2.6)$ & $72.8(2.9)$ & $73.4(2.7)$ & & & & & & \\
\hline $\begin{array}{l}\text { Snellen } \\
\text { equivalent }\end{array}$ & $20 / 32$ & $20 / 40$ & $20 / 40$ & & & & & & \\
\hline \multicolumn{10}{|c|}{ Visual acuity at 1 year } \\
\hline $\begin{array}{l}\text { Mean (SD) } \\
\text { letter score } \\
\text { Approximate }\end{array}$ & $81.4(8.3)$ & $79.9(10.1)$ & $81.6(6.8)$ & & & & & & \\
\hline $\begin{array}{l}\text { Snellen } \\
\text { equivalent }\end{array}$ & $20 / 25$ & $20 / 25$ & $20 / 25$ & & & & & & \\
\hline \multicolumn{10}{|c|}{ Change from baseline in letter score } \\
\hline $\begin{array}{l}\text { Mean (SD) } \\
\text { improvement }\end{array}$ & $8.0(7.6)$ & $7.5(7.4)$ & $8.3(6.8)$ & $0.7(-1.3$ to 2.7$)$ & 0.69 & $\begin{array}{c}-0.4(-2.3 \text { to } \\
1.5)\end{array}$ & 0.69 & $\begin{array}{c}1.1(-0.9 \text { to } \\
3.1)\end{array}$ & 0.69 \\
\hline $\begin{array}{l}\text { Improvement } \\
\text { of } \geq 10 \text { letters, } \\
n(\%)\end{array}$ & $53(50)$ & $47(45)$ & $52(50)$ & $6(-9$ to 21$)$ & 0.82 & $0(-13$ to 14$)$ & 0.95 & $6(-10$ to 21$)$ & 0.82 \\
\hline $\begin{array}{l}\text { Worsening of } \\
\geq 10 \text { letters, } n \\
(\%)\end{array}$ & $4(4)$ & $2(2)$ & $1(1)$ & $2(-3$ to 6$)$ & 0.54 & $3(-1$ to 7$)$ & 0.54 & $-1(-4$ to 2$)$ & 0.54 \\
\hline $\begin{array}{l}\text { Improvement } \\
\text { of } \geq 15 \text { letters, } \\
n(\%)\end{array}$ & $19(18)$ & $17(16)$ & $16(15)$ & $2(-7$ to 11$)$ & 0.73 & $4(-5$ to 12$)$ & 0.73 & $-2(-10$ to 7$)$ & 0.73 \\
\hline $\begin{array}{l}\text { Worsening of } \\
\geq 15 \text { letters, } n \\
(\%)\end{array}$ & $2(2)$ & $1(1)$ & $1(1)$ & $1(-2$ to 4$)$ & 0.99 & $1(-2$ to 4$)$ & 0.99 & $0(-3$ to 3$)$ & 0.99 \\
\hline
\end{tabular}

${ }^{a}$ Treatment group comparisons were performed with ANCOVA models adjusted for continuous baseline visual acuity or from binomial regression models adjusted for categorical baseline visual acuity (adapted from Diabetic Retinopathy Clinical Research Network et al. [29]). CI: confidence interval; SD: standard deviation; $n=$ number.

and a combination of ranibizumab $0.5 \mathrm{mg}$ and laser (baseline and month 3) [43]. The results of this study found that, at month 6 , BCVA improvement was significantly greater in those eyes receiving ranibizumab alone $(p=0.01)$.

The RESOLVE was a 12-month, multicenter, shamcontrolled, double-masked study designed to evaluate the efficacy and safety of ranibizumab in DME [44]. Study patients were randomized to ranibizumab $(0.3$ or $0.5 \mathrm{mg}$; $n=51$ each $)$ or sham $(n=49)$ [44]. The mean average change in BCVA from month 1 to month 12 was significantly greater with ranibizumab (7.8 letters) than with sham $(-0.1$ letters) $(p<0.0001)$. Similarly, the mean change in CMT was significantly greater with ranibizumab $(-194.2 \mu \mathrm{m})$ than with sham $(-48.4 \mu \mathrm{m})(p<0.001)$ [44]. Regarding the safety profile, there were no significant differences in the incidence of serious and nonserious ocular/nonocular adverse events between ranibizumab and sham. In both arms (without significant differences between them), the most frequently reported adverse events were conjunctival hemorrhage, ocular hypertension, and eye pain [44].

The RESTORE study was a 12-month, multicenter, prospective, randomized, double-masked, and laser-controlled phase III study that evaluated the efficacy (in terms of BCVA improvement) of ranibizumab $0.5 \mathrm{mg}+$ sham laser vs. ranibizumab + laser vs. laser alone [45]. As compared to laser, ranibizumab alone achieved a greater BCVA improvement $(p<0.0001)$ and a greater proportion of gaining $\geq 15$ letters $(p=0.0005)$. CMT reduction was significantly 
greater with ranibizumab alone than with laser alone $(p<0.0001)$. There were no significant differences in either BCVA improvement or CMT reduction between ranibizumab alone and ranibizumab + laser. Regarding the safety profile, there was no significant association between ranibizumab (alone or in combination) and the incidence of cardiovascular or cerebrovascular events [45].

Based on the RESTORE results, the European Medicines Agency (EMAs) approved in 2011 the use of ranibizumab for the treatment of DME [6]. Interestingly, the dose approved by the EMAs $(0.5 \mathrm{mg})$ differs from those approved by the Food and Drug Administration (0.3 mg) [6].

The RISE and the RIDE are two multicenter, prospective, randomized, and sham injection-controlled studies that evaluated the efficacy and safety of ranibizumab in DME patients [46]. Seven hundred and fifty-nine patients (377 in RISE and 382 in RIDE) were randomized to receive monthly sham injections or intravitreal injections of ranibizumab $0.3 \mathrm{mg}$ or $0.5 \mathrm{mg}$. The proportion of patients gaining $\geq 15$ letters in RISE was the $18.1 \%, 39.2 \%$, and $44.8 \%$ in eyes treated with sham, ranibizumab $0.5 \mathrm{mg}$, and ranibizumab $0.3 \mathrm{mg}$, respectively $(p<0.0001$ between sham and $0.3 \mathrm{mg}$ and $p=0.0002$ between sham and $0.5 \mathrm{mg}$ ) [46]. Regarding RIDE, a greater proportion of patients achieved a BCVA improvement $\geq 15$ in the ranibizumab $0.3 \mathrm{mg}$ group $(33.6 \%$, $p<0.0001$ vs. sham) and in the ranibizumab $0.5 \mathrm{mg}$ group $(45.7 \%, p<0.0001$ vs. sham) than in the sham-treated group (12.3\%). BCVA improvements were paralleled by rapid reductions in CMT. Independently of the study (RISE or RIDE) and the ranibizumab dose ( 0.3 or 0.5 ), the mean CMT reduction was significantly greater in ranibizumab groups than in sham groups $(p<0.0001$ each) [46]. Safety profile showed that serious adverse events were uncommon and included one case of endophthalmitis in RISE and 3 in RIDE and 2 cases of traumatic cataract in RISE and one in RIDE. Systemic adverse events potentially related to systemic VEGF inhibitions occurred in $10.6 \%$ and $9.4 \%$ of shamtreated patients in RISE and RIDE, respectively, and in 5.6\% and $1.9 \%$ of ranibizumab-treated eyes (both doses) across the studies [46].

The 3-year follow-up results of the study that evaluated the effect of prompt vs. deferred ( $\geq 24$ weeks) of laser treatment in eyes receiving intravitreal injections of intravitreal ranibizumab $(0.5 \mathrm{mg})$ suggested that, in eyes with DME, deferring laser $\geq 24$ weeks provided better functional outcomes than prompt laser treatment [47]. However, this finding might be justified by the fact that eyes that received prompt laser received fewer ranibizumab injections than needed [47].

The extension from 24 to 36 months of the READ study found that, in the ranibizumab group, the variation in BCVA from month 24 to month 36 was +3.1 letters $(p=0.009)$ and foveal thickness was thinner at month 36 than at month 24 (mean variation -70 microns, $p=0.006$ ) [48]. The results of this study found that although long-term functional and anatomical outcomes with ranibizumab were very good, many patients required frequent injections to achieve such outcomes [48].
The long-term (three years) outcomes of the RISE and RIDE studies were published in 2013 [49]. RISE and RIDE were two phase III, multicenter clinical trials that were sham-controlled for two years. Patients were randomly assigned in a 1:1 regime to monthly sham injection or intravitreal ranibizumab (either 0.3 or $0.5 \mathrm{mg}$ ) during a follow-up period of two years. In the third year, sham patients, while still masked, were eligible to crossover to monthly $0.5 \mathrm{mg}$ ranibizumab [49]. Functional outcomes at month 36 observed in the ranibizumab groups were in line with those previously reported in the two-year follow-up study [46]. The proportion of patients achieving a gaining $\geq 15$ letters in BCVA was greater, in both RISE and RIDE, in the ranibizumab groups (independently of the dose) than in the sham group [49]. Patients who previously received sham when were changed to ranibizumab 0.5 showed lower BCVA improvements than those obtained by ranibizumab after their first year of treatment [49]. The incidence of serious adverse events potentially related to systemic VEGF inhibition was $19.7 \%$ and $16.8 \%$ in eyes treated with ranibizumab $0.3 \mathrm{mg}$ and $0.5 \mathrm{mg}$, respectively [49].

The RESTORE extension evaluated the results of the protocol RESTORE after three years of follow-up. The RESTORE had a 12-month double-masked phase and a 24month open-label extension [50]. 79\% (240/303) of the patients who completed the first phase of the RESTORE were included in the extension, and 208 patients (86.7\%) completed the extension study. In those patients previously treated with ranibizumab during the 12-month doublemasked phase, BCVA improvement and CMT reduction were maintained. In the laser group, when ranibizumab intravitreal injections were allowed, there were a BCVA improvement $(+6.0$ letters $)$ and a retinal thickness reduction $(-142.7 \mu \mathrm{m})$ at month 36. Cataract, with an incidence of $16.3 \%$, was the most frequently reported adverse event. Eight patients died during the study; none were suspected to be related to the study drug/procedure [50].

The LUCIDATE study was a prospective, randomized, single-masked, and single-center clinical trial that compared the functional and anatomical effects of laser versus ranibizumab in DME patients [51]. Patients were randomly assigned in a $2: 1$ ratio to 3 monthly doses of ranibizumab and retreatment when needed vs. laser at baseline and repeated every 12 weeks (as needed) [51]. Mean BCVA improvement was +6.0 letters in the ranibizumab group vs. -0.9 letters in the laser group. Additionally, retinal sensitivity and electrophysiology function were improved with ranibizumab. Anatomic outcomes were better in the ranibizumab group than in the laser group. No safety issues were reported [51].

The 5-year results of the DRCR.net study evaluating ranibizumab plus prompt or deferred laser or triamcinolone plus prompt laser for diabetic macular edema suggested that, in eyes with DME, focal/grid laser treatment at the initiation of intravitreal ranibizumab treatment did not provide better results than deferring laser treatment for $\geq 24$ weeks [52]. Additionally, deferring laser was also associated with an increase in the number of intravitreal injections of ranibizumab [52]. 
The REVEAL study was a 12-month, randomized, double-masked, multicenter, laser-controlled, phase III study conducted in the Asian population that compared the efficacy of three different therapeutic strategies: ranibizumab + sham laser, ranibizumab + active laser, or sham injection + active laser [53]. Ranibizumab alone or in combination with laser provided better functional and anatomic outcomes than laser alone. The most frequently reported ocular adverse event was conjunctival hemorrhage. There was no evidence of adverse events associated with systemic inhibition of VEGF [53].

The RELIGHT study evaluated the potential benefits of tailoring the ranibizumab treatment regime to the DME patient's needs [54]. Patients received initially a loading dose of 3 initial intravitreal ranibizumab injections (baseline and months 1 and 2). Based on individualized BCVA and CMT, patients received monthly intravitreal ranibizumab injections (months 3 to 5) and bimonthly between months 6 and 18 [54]. The results of this study suggested that functional outcomes obtained during the initial 6-month treatment regime were maintained during the bimonthly tailored treatment [54].

Similarly, the RETAIN study was designed to evaluate whether an incremental extension of intertreatment intervals ( 1 to 3 months) would be feasible [55]. In terms of functional outcomes, the incremental extension regimen did not provide worse results than a standard pro re nata (PRN) regime (monthly followed and treated according to signs of disease activity). However, the number of intravitreal injections was slightly greater with the incremental extension regimen [55].

The READ-3 study was a multicenter, prospective, and randomized study that compared the efficacy of intravitreal injections of ranibizumab $2.0 \mathrm{mg}$ with ranibizumab $0.5 \mathrm{mg}$ in eyes with DME [56]. The results of this study suggested that BCVA improvement was not superior with ranibizumab $2.0 \mathrm{mg}$ than with ranibizumab $0.5 \mathrm{mg}$. The safety profile of both doses was similar [56].

The open-label extension of the RISE and RIDE protocols tried to answer the question of whether an intravitreal ranibizumab "less-than-monthly injection" regime would be as effective as monthly injection regime [57]. According to the results of this study, the functional and anatomic outcomes achieved with monthly ranibizumab might be maintained with a reduction in treatment frequency [57].

The efficacy and safety of ranibizumab and bevacizumab were compared directly in a randomized, doublemasked, 36-week, and 3-period crossover study [58]. Patients received monthly intravitreal injections of bevacizumab $(1.25 \mathrm{mg})$ or ranibizumab $(0.3 \mathrm{mg})$. The results of this study found a slightly but statistically significant difference in BCVA improvement (difference of 1.3 letters in favor of ranibizumab; $p=0.039$ ) and in the mean CMT reduction (difference of $48 \mu \mathrm{m}$ in favor of ranibizumab; $p<0.001)$ [58].

The question of whether early visual acuity response to ranibizumab in DME patients is associated with long-term outcomes was evaluated in a post hoc analysis of the DRCR. net Protocol I [59]. The results of this study suggested that a poor early response was associated with a poor long-term visual outcome. Additionally, this study found that $39.7 \%$ of the eyes underwent ranibizumab (with or without laser) did not adequately respond (BCVA improvement $<5$ letters) at week 12 [59].

The two-year effectiveness of intravitreal ranibizumab in combination with a nutritional supplement rich in docosahexaenoic acid plus antioxidants was evaluated in a prospective, randomized, and single-blind controlled study [60]. At month 24 , the combined therapy provided a statistically significant CMT reduction as compared with intravitreal ranibizumab alone. However, there were no significant differences in BCVA improvement between the two strategies [60].

The TREX-DME study was a multicenter, prospective, and randomized clinical trial designed for comparing monthly ranibizumab injections vs. an incremental extension algorithm [61]. The results of this study suggested that the incremental extension strategy did not provide worse functional or anatomic outcomes, while decreasing the number of injections administered [61].

The ROTATE trial was a prospective and open-label study that evaluates the efficacy of intravitreal of ranibizumab $0.3 \mathrm{mg}$ in eyes with persistent DME after intravitreal bevacizumab treatment [62]. The results of this study showed that the mean BCVA was significantly improved (+6.5 letters) and CMT was significantly reduced $(-116 \mu \mathrm{m})$ under intravitreal ranibizumab treatment. On the negative side, systemic adverse events included two deaths, stroke, and myocardial infarction [62].

The RELATION study, a prospective, double-masked, multicenter phase IIIb trial, assessed the efficacy and safety of intravitreal ranibizumab $0.5 \mathrm{mg}$ plus laser versus laser monotherapy in patients with DME [63]. As compared with laser monotherapy, ranibizumab + laser provided a significantly greater BCVA improvement (mean difference between groups, 4.2 letters; $p=0.001$ ). However, there was no significant difference in CMT reduction between the two groups $(p=0.28)[63]$.

The REFINE was a phase III, 12-month, doublemasked, multicenter, laser-controlled study conducted on Chinese patients with DME. Patients were randomly assigned (4.1) to receive intravitreal ranibizumab injections or laser [64]. The mean BCVA improvement with ranibizumab at month 12 (+7.9 letters) was statistically significantly greater $(p<0.001)$ than that observed with laser (+2.5 letters) [64].

Table 3 summarizes the main results of ranibizumab observed in different studies included in this review.

(1) Safety. As regards the safety profile, the incidence and characteristics of the adverse events were similar to that observed with bevacizumab. Systemic VEGF inhibitionrelated adverse events such as stroke and myocardial infarction have been described with the administration of ranibizumab [45-64]. The most commonly reported ocular adverse event among the different studies was conjunctival hemorrhage. Other ocular side effects included endophthalmitis, ocular hypertension, and retinal detachment [45-64]. 
TABLE 3: Overview of the functional and anatomic results of ranibizumab.

\begin{tabular}{|c|c|c|c|c|c|c|c|c|}
\hline \multirow{2}{*}{ Study } & \multirow{2}{*}{ Ref. } & \multirow{2}{*}{ Duration (w) } & \multirow{2}{*}{ Regimen } & \multirow{2}{*}{$N$ (eyes) } & \multicolumn{2}{|c|}{ BCVA (ETDRS letters) } & \multicolumn{2}{|c|}{ CRT $(\mu \mathrm{m})$} \\
\hline & & & & & Baseline & Change & Baseline & Change \\
\hline \multirow{3}{*}{ READ-2 } & \multirow{3}{*}[43]{} & \multirow{3}{*}{24} & Rani & 42 & 24.85 & $7.24^{* *}$ & 422 & -106.3 \\
\hline & & & Rani + laser & 42 & 24.87 & 3.8 & 474.5 & -117.2 \\
\hline & & & Laser & 42 & 28.35 & -0.43 & 439.6 & -82.8 \\
\hline \multirow{2}{*}{ RESOLVE } & \multirow{2}{*}[44]{} & \multirow{2}{*}{52} & Rani & 102 & $60.2(9.9)$ & $7.8(7.7)^{* *}$ & $455.4(114.2)$ & $-194.2(135.1)^{* *}$ \\
\hline & & & Sham & 49 & $61.1(9.0)$ & $-0.1(9.8)$ & $448.9(102.8)$ & $-48.4(153.4)$ \\
\hline \multirow{3}{*}{ RESTORE } & \multirow{3}{*}[45]{} & \multirow{3}{*}{52} & Rani + sham & 116 & N.A. & $6.1(6.3)^{* *}$ & N.A. & $-118.7(115.1)^{* *}$ \\
\hline & & & Rani + laser & 118 & N.A. & $5.9(7.9)^{* *}$ & N.A. & $-128.3(114.3)^{* *}$ \\
\hline & & & Laser + sham & 111 & N.A. & $0.8(8.6)$ & N.A. & $-61.3(132.3)$ \\
\hline \multirow{3}{*}{ RESTORE Ext. } & \multirow{3}{*}[55]{} & \multirow{3}{*}{156} & Rani + sham & 116 & N.A. & $8.0(1.1)$ & 116 & -142.1 \\
\hline & & & Rani + laser & 118 & N.A. & $6.7(1.1)$ & 118 & -145.9 \\
\hline & & & Laser + sham & 111 & N.A. & $6.0(1.1)$ & 111 & -142.7 \\
\hline \multirow{3}{*}{ DRCR.net } & \multirow{3}{*}[47]{} & \multirow{3}{*}{56} & Rani 0.5 & 113 & $68(56--75)$ & $+2(-3 \text { to }+7)^{* *}$ & $352(283--476)$ & $-26(-92 \text { to }+15)^{*}$ \\
\hline & & & Triam & 109 & $67(59--75)$ & $+1(-3 \text { to }+8)^{* *}$ & $359(271--472)$ & $-75(-168 \text { to }-17)^{* *}$ \\
\hline & & & Sham & 123 & $67(52--75)$ & $-2(-8$ to +3$)$ & $355(285--510)$ & $0(-80$ to +70$)$ \\
\hline \multirow{3}{*}{ RISE } & \multirow{3}{*}[46]{} & \multirow{3}{*}{104} & Rani $0.3 \mathrm{mg}$ & 125 & $54.7(12.6)$ & $12.5^{* * *}$ & 4745. (174.8) & $-250.6^{* * *}$ \\
\hline & & & Rani $0.5 \mathrm{mg}$ & 125 & $56.9(11.6)$ & $11.9^{* * *}$ & $463.8(144.0)$ & $-253.1^{* * *}$ \\
\hline & & & Sham & 127 & $57.2(11.1)$ & 2.6 & $467.3(152.0)$ & -133.4 \\
\hline \multirow{3}{*}{ RIDE } & \multirow{3}{*}[46]{} & & Rani $0.3 \mathrm{mg}$ & 125 & $57.5(11.6)$ & $10.9^{* * *}$ & $482.6(149.3)$ & $-259.8^{* * *}$ \\
\hline & & 104 & Rani $0.5 \mathrm{mg}$ & 127 & $56.9(11.8)$ & $12.0^{* * *}$ & $463.8(175.5)$ & $-270.7^{* * *}$ \\
\hline & & & Sham & 130 & $57.3(11.2)$ & 2.3 & $447.4(154.4)$ & -125.8 \\
\hline & & & Rani $0.3 \mathrm{mg}$ & 125 & $54.7(12.6)$ & $14.2(12.8)^{* * *}$ & 4745. (174.8) & $-261.2(196.5)$ \\
\hline RISE & [49] & 156 & Rani $0.5 \mathrm{mg}$ & 125 & $56.9(11.6)$ & $11.0(12.9)^{* * *}$ & $463.8(144.0)$ & $-269.1(178.9)$ \\
\hline & & & Sham & 127 & $57.2(11.1)$ & $4.3(14.9)$ & $467.3(152.0)$ & $-200.1(215.6)$ \\
\hline & & & Rani $0.3 \mathrm{mg}$ & 125 & $57.5(11.6)$ & $10.6(12.9)^{* * *}$ & $482.6(149.3)$ & $-261.8(180.8)$ \\
\hline RIDE & {$[49]$} & 156 & Rani $0.5 \mathrm{mg}$ & 127 & $56.9(11.8)$ & $11.4(16.3)^{* * *}$ & $463.8(175.5)$ & $-266.7(207.8)$ \\
\hline & & & Sham & 130 & $57.3(11.2)$ & $4.7(13.3)$ & $447.4(154.4)$ & $-213.2(193.5)$ \\
\hline & & & Rani $0.3 \mathrm{mg}$ & 89 & $54.4(12.0)$ & $-1.7(-3.6$ to 0.2$)$ & $475.9(170.2)$ & $23.3(-7.7$ to 54.3$)$ \\
\hline RISE & {$[57]$} & 208 & Rani $0.5 \mathrm{mg}$ & 79 & $56.5(10.9)$ & $0.8(-1.1$ to 2.7 & $476.7(139.5)$ & $4.2(-17.1$ to 25.4$)$ \\
\hline & & & Sham & 77 & $57.8(10.5)$ & $1.3(-0.3$ to 2.9$)$ & $462.8(141.4)$ & $29.6(3.4-55.7)$ \\
\hline & & & Rani $0.3 \mathrm{mg}$ & 83 & $58.3(11.3)$ & $-0.9(-3.6$ to 1.8$)$ & $480.3(186.9)$ & 46.1 ( -2.6 to 94.8 \\
\hline RIDE & {$[57]$} & 208 & Rani $0.5 \mathrm{mg}$ & 84 & $56.9(11.8)$ & $0.6(-1.2$ to 2.4 & $481.1(163.2)$ & $44.1(16.1-72.1)$ \\
\hline & & & Sham & 88 & $57.8(11.4)$ & $-2.6(-5.6$ to 0.5$)$ & $441.3(146.3)$ & $9.6(-18.4$ to 37.6$)$ \\
\hline REFINE & {$[64$} & 52 & Rani $0.5 \mathrm{mg}$ & 307 & $59.6(10.5)$ & $7.8(0.7)^{* *}$ & $473.4(166.1)$ & $-146.5(157.6)^{* *}$ \\
\hline REFINE & [64] & 52 & Laser & 77 & $58.2(9.4)$ & $2.5(7.8)$ & $475.0(161.5)$ & $-85.9(166.6)$ \\
\hline
\end{tabular}

Note. READ-2: measured subfoveal thickness. RESOLVE: ranibizumab group included intravitreal injections of $0.3 \mathrm{mg}$ and $0.5 \mathrm{mg}$ ( 51 eyes each). DRCR.net: patients received laser besides their treatment assigned; measured central subfield thickness; differences were calculated at week 14 (primary outcome of the study). RESTORE extension: all patients enrolled in the extension study were eligible to receive intravitreal ranibizumab $0.5 \mathrm{mg}$ injections (subgroups were maintained for evaluating the effect of ranibizumab according to the initial treatment). ${ }^{*} p<0.01$ vs. reference comparator (laser, sham, triamcinolone, etc.). ${ }^{* *} p<0.001$ vs. reference comparator (laser, sham, triamcinolone, etc.). ${ }^{* * *} p<0.0001$ vs. reference comparator (laser, sham, triamcinolone, etc.). w: weeks; BCVA: best-corrected visual acuity; EDTRS: Early Treatment of Diabetic Retinopathy Study; CRT: central retina thickness; DRCR.net: Diabetic Retinopathy Clinical Research Network; Rani: ranibizumab; Triam: triamcinolone acetonide.

Repeated intravitreal ranibizumab injections may increase the risk of ocular hypertension [65]. According to the results of the DRCR.net study, repeated intravitreal injections of ranibizumab were associated with a greater probability of sustained intraocular pressure (IOP) elevation than laser treatment (hazard ratio 2.9, $p=0.01$ ) [65].

Regarding the potential negative effect on corneal endothelium of ranibizumab or bevacizumab, monthly intravitreal 0.5 ranibizumab or $1.25 \mathrm{mg}$ bevacizumab during three months did not show any negative effect on corneal endothelium [66].

3.1.4. Aflibercept. Aflibercept (EYLEA ${ }^{\circledR}$; Bayer HealthCare, Berlin, Germany/Regeneron Pharmaceuticals Inc., Tarrytown,
NY, USA) is a fusion protein $(115 \mathrm{kDa})$ comprising the second Ig domain of human VEGFR1, the third Ig domain of human VEGFR2, and the Fc region of a human IgG1 [67-69].

The first high-quality scientific evidence published about the role of aflibercept in the management of DME were the VIVID in Europe [70] and the VISTA in the United States [71].

VIVID and VISTA were two similarly designed randomized phase III trials that compared the efficacy and safety of two doses of intravitreal regimes of aflibercept vs. laser for DME treatment [70, 71]. After a monthly loading dose of 5 injections, aflibercept $2 \mathrm{mg}$ was administered every 4 (IA4W) or every 8 weeks (IA8W) [70, 71]. After 100 weeks of follow-up, the proportion of patients achieving a BCVA improvement of $\geq 15$ letters in VIVID was $38.2 \%, 31.1 \%$, and 
$12.1 \%$ for the IA $4 \mathrm{~W}$, IA8W, and laser treatment regimes, respectively $(p<0.0001)$, and in VISTA was $38.3 \%, 33.1 \%$, and $13.0 \%$ for the IA4W, IA8W, and laser treatment regimes, respectively $(p<0.0001)$. The pooled mean BCVA improvement from baseline to week 100 was 10.7 and 10.3 for IA4W and IA8W, respectively. There were no significant differences in terms of BCVA improvement between IA4W and IA8W regimes [70, 71].

Based on these results, the European Medicines Agency (EMA), in 2014, and the Food and Drug Administration (FDA), in 2015, approved the use of aflibercept for treating DME. FDA approved a dose of $2 \mathrm{mg}$ per injection (5 monthly injections as loading dose plus bimonthly injections thereafter), while EMA adds (to the aforementioned) the option to establish an incremental extension of intertreatment intervals after the first year of treatment [6].

A post hoc analysis of the VIVID and VISTA trials compared the effect of intravitreal aflibercept on functional and anatomic outcomes in DME patients with and without prior anti-VEGF treatment [72]. Mean BCVA improvement at week 100 in those eyes that did not receive previous antiVEGF treatment was 12.0, 11.3, and +2.1 letters for the IA4W, IA8W, and laser treatment regimes, respectively. In previously treated eyes, mean BCVA improvements at week 100 were $+10.9,+10.8$, and -0.8 letters. At week 100 , mean CMT reductions in previously treated eyes were $180.1 \mu \mathrm{m}$, $196.4 \mu \mathrm{m}$, and $94.1 \mu \mathrm{m}$ for the IA4W, IA8W, and laser treatment regimes, respectively. In eyes without previous anti-VEGF treatment, at week 100, mean CMT reductions were $200.0 \mu \mathrm{m}, 186.7 \mu \mathrm{m}$, and $76.9 \mu \mathrm{m}$ for the IA4W, IA8W, and laser treatment regimes, respectively [72]. Functional and anatomic improvements were statistically significant with both intravitreal aflibercept regimes as compared with laser. There were no statistically significant differences in both functional and anatomic outcomes between the two intravitreal aflibercept regimes [72].

The ENDURANCE extension study was a phase IV, open-label study conducted on patients who completed the VIVID and VISTA DME trials [73]. During the ENDURANCE study, both interval between patient visits and intravitreal aflibercept injections were tailored according to the patient's needs [73]. Sixty patients were enrolled in the ENDURANCE study. The BCVA improvements achieved during the VISTA were maintained and stable $(<1.5$ letters $)$ over the 12-month follow-up.

Similar to BCVA, mean CMT remained relatively stable during the ENDURANCE study [73]. Regarding treatment needs, $42(70 \%)$ received $\geq 1$ intravitreal injection of aflibercept (mean 4.5 injections), without any significant impact of the treatment received during the VISTA [73].

The results of the 148-week analysis from the VISTA and VIVID studies confirmed the previous findings [74]. BCVA improvements achieved with both intravitreal aflibercept regimes at week 52 and week 100 were maintained at week 148. As regards the safety profile, the findings were consistent with the previous reports [70-73].

A post hoc analysis of Protocol $T$ found that, for those eyes with a baseline visual acuity $<69$ letters, the BCVA improvement achieved with aflibercept was statistically greater than that obtained with bevacizumab and ranibizumab at 1 year. However, at 2 years, aflibercept was only superior, in terms of BCVA improvement, to bevacizumab [75]. Regarding retinal thickness, in eyes with baseline visual acuity <20/50, reduction observed at 1 year with bevacizumab was lower than with the other anti-VEGF drugs, but at 2 years the differences had diminished [75].

A secondary analysis of the Protocol $\mathrm{T}$ compared changes in diabetic retinopathy severity during aflibercept, bevacizumab, or ranibizumab treatment for DME [76]. At 1 year, in eyes with no proliferative diabetic retinopathy, a significantly greater proportion of patients treated with aflibercept or ranibizumab had improvement in diabetic retinopathy severity as compared with bevacizumab ( $p=0.004$ for aflibercept vs. bevacizumab and $p=0.01$ for ranibizumab vs. bevacizumab), but there was no difference between aflibercept vs. ranibizumab $(p=0.51)$ [76]. However, at 2 years, no treatment group differences were identified in the proportion of patients who had diabetic retinopathy improvement. As regards the eyes with diabetic retinopathy, at 1 year a significantly greater proportion of patients treated with aflibercept had improvement in diabetic retinopathy severity as compared with bevacizumab $(p<0.001)$ or ranibizumab $(p=0.02)$, but not between ranibizumab and bevacizumab $(p=0.09)$ [76].

Unlike eyes with no proliferative diabetic retinopathy, these rates and treatment group differences seemed to be maintained at 2 years [76].

The 2-year outcomes of the ENDURANCE extension study found that the number of intravitreal aflibercept injections was substantially reduced in the fourth and fifth years of aflibercept dosing following initiation of therapy in the VISTA DME trial [77]. BCVA improvements achieved during the 3-year VISTA trial were maintained [77].

An additional post hoc analysis of the Protocol $\mathrm{T}$ evaluated the proportion of eyes with persistent DME after 24 weeks of treatment with aflibercept, bevacizumab, or ranibizumab. Persistent DME through 24 weeks was significantly less frequent with aflibercept than with the other treatments $(p<0.001$ vs. bevacizumab and $p=0.05$ vs. ranibizumab) and less frequent with ranibizumab than with bevacizumab $(p<0.001)$ [78]. Although the proportion of eyes with persistent DME was significantly lower with aflibercept, $31.6 \%$ of the eyes (60/190) did not adequately respond to this treatment [78].

An integrated post hoc subanalysis of the two phase II trials VISTA and VIVID assessed the effect of baseline factors on differences in BCVA improvement with intravitreal aflibercept injection vs. laser in DME patients [79]. According to the results of this study, BCVA improvement was significantly greater with aflibercept than with laser and was not influenced by any baseline factor [79].

The Protocol V was a prospective and randomized clinical trial that compared three different strategies for treating eyes with DME and good visual acuity (20/25 or better) [80]. Study eyes were randomly assigned in a $1: 1: 1$ ratio to $2.0 \mathrm{mg}$ of aflibercept, focal/grid laser photocoagulation, or observation. In the laser photocoagulation and observation groups, it was allowed to start with aflibercept if 
visual acuity met specific worsening criteria. At 2 years, there were no significant differences in the proportion of eyes with at least a 5-letter visual acuity decrease (aflibercept vs. laser, $p=0.79$; aflibercept vs. observation, $p=0.79$; and laser vs. observation, $p=0.79$ ). In other words, in eyes with DME and good visual acuity, aflibercept or laser photocoagulation appeared to be no superior to observation [80].

The real-world functional and anatomic outcomes of intravitreal aflibercept in DME patients, either naïve or previously treated, were assessed in a prospective, observational, and multicenter cohort study conducted in France [81]. The APOLLON evaluated, as the primary outcome, the mean change in BCVA from baseline to month 12. The study included 147 patients (77 treatment-naïve and 70 previously treated) followed up for at least 12 months. The mean improvement in BCVA at month 12 was $7.8 \pm 12.3$ and $5.0 \pm 11.3$ letters in treatment-naïve and previously treated patients, respectively, $p=0.1541$ (independent-sample Student's $t$-test) [81]. Intravitreal aflibercept significantly reduced CMT in both groups, without differences between them. The mean intravitreal injection administered during the study was $7.6 \pm 2.5$ in the treatment-naïve group and $7.6 \pm 2.3$ in the previously treated one, $p=1.000$ (independent-sample Student's $t$-test) [81].

Table 4 summarizes the main results of ranibizumab observed in different studies included in this review.

(1) Safety. The incidence of either ocular or systemic side effects did not significantly differ from those reported for bevacizumab or ranibizumab [35, 36, 70-81].

Ziv-aflibercept (Zaltrap, Sanofi-Aventis US, LLC, Bridgewater, New Jersey, USA, and Regeneron Pharmaceuticals, Inc., Tarrytown, New York, USA), a recombinant fusion protein, has a mechanism that is similar in action to that of aflibercept and is available at a lower cost than the proprietary anti-vascular endothelial growth factor (VEGF) drug $[82,83]$.

The results of a 3-month prospective study, which included 17 eyes with DME, found that off-label use of intravitreal ziv-aflibercept improved visual acuity, without detectable ocular toxicity or systemic side effects in DME [84].

Additionally, the results of a retrospective study that evaluated the clinical outcomes and safety profile of zivaflibercept in eyes that received $\geq 10$ intravitreal injections found that multiple intravitreal injections of ziv-aflibercept were associated with a significant improvement in both functional and anatomic outcomes, with a good safety profile [85].

3.1.5. Conbercept. Conbercept is a recombinant soluble VEGF receptor decoy [86]. Its affinity for VEGF is 50 times that of bevacizumab and 30 times that of ranibizumab [87].

A retrospective study compared the efficacy of intravitreal conbercept either alone or in combination with laser [88]. The results of this study suggested that both treatment strategies significantly improved BCVA and reduced CMT, without differences between them $(p=0.164$ and $p=0.149$ for the BCVA and CMT, respectively) [88]. Nevertheless, the number of intravitreal injections of conbercept was significantly lower with the combined therapy $(3.3 \pm 1.2$ per eye) than with conbercept alone (5.6 \pm 0.8 per eye), $p<0.001$ [88].

The efficacy of intravitreal conbercept and ranibizumab for treating DME was evaluated in a 12-month, retrospective, and real-life study [89]. Patients received intravitreal conbercept injections or intravitreal ranibizumab injections, once a month for 3 months followed by as-needed therapy. At month 12 , BCVA improvement was $9.3 \pm 5.2$ and $8.9 \pm 4.4$ in the conbercept and ranibizumab groups, respectively, $p<0.001$ each (with no significant differences between groups, $p=0.756$ ). At month 12 , the mean CMT reduction was $138.4 \pm 97.7 \mu \mathrm{m}$ in the conbercept group and $145.2 \pm 72.5 \mu \mathrm{m}$ in the ranibizumab group, $p<0.001$ each (with no significant differences between groups, $p=0.748$ ) [89].

The efficacy and safety of intravitreal conbercept for the treatment of DME were evaluated in a retrospective study. The BCVA improvement at months 1 and 3 was sadistically significant; however, such improvement started to decrease at month 6 [90]. Notably, 32.6\% of the eyes treated with intravitreal conbercept were not sensitive to it within half a year. CMT reduction was basically maintained at month 12 [90].

Moreover, a meta-analysis that compared the efficacy of conbercept and ranibizumab for the treatment of DME reported that intravitreal conbercept was significantly superior to ranibizumab in terms of CMT reduction, but no statistically significant difference with regard to visual improvement [91].

Additionally, it appears that conbercept was able to significantly improve the BCVA independently of the baseline visual acuity, although for worse baseline visual acuity (20/50 or worse), BCVA improvement was more prominent than that of better baseline visual acuity (20/32 to 20/40) subgroup [92].

The results of a meta-analysis, which included 588 patients, compared the effect and safety of conbercept and ranibizumab in the treatment of DME suggested that intravitreal injections of conbercept were superior to ranibizumab in both reducing CRT and improving BCVA [93]. Regarding safety, the pooled results showed that there was no significant difference in the risk of intraocular pressure increase (or conjunctival hemorrhage between two groups [93].vd

(1) Safety. The most frequently reported ocular adverse event was conjunctival hemorrhage [88-93]. The incidence and type of adverse events did not significantly differ from those previously reported for other anti-VEGF therapies (Sections 3.1.2 to 3.1.4).

3.2. Steroids. Since there is increasing evidence about the role of inflammation on the pathophysiology of DME, corticosteroids have taken an active role in its treatment [8-11]. Corticosteroid therapy is able to inhibit many of the processes known to be involved in the progression of DME, 
TABLE 4: Overview of the functional and anatomic results of aflibercept.

\begin{tabular}{|c|c|c|c|c|c|c|c|c|}
\hline \multirow{2}{*}{ Study } & \multirow{2}{*}{ Ref. } & \multirow{2}{*}{ Duration (w) } & \multirow{2}{*}{ Regimen } & \multirow{2}{*}{$N$ (eyes) } & \multicolumn{2}{|c|}{ BCVA (ETDRS letters) } & \multicolumn{2}{|c|}{ CRT $(\mu \mathrm{m})$} \\
\hline & & & & & Baseline & Change & Baseline & Change \\
\hline \multirow{9}{*}{ VISTA } & \multirow{3}{*}[70]{} & \multirow{3}{*}{52} & IA4W & 154 & $58.9(10.8)$ & $12.5^{* * *}$ & $485(157)$ & $-185.9^{* * *}$ \\
\hline & & & IA8W & 151 & $59.4(10.9)$ & $10.7^{* * *}$ & $479(154)$ & $-183.1^{* * *}$ \\
\hline & & & Laser & 154 & $59.7(10.9)$ & 0.2 & $483(153)$ & -73.3 \\
\hline & \multirow{3}{*}[70]{} & \multirow{3}{*}{100} & IA $4 \mathrm{~W}$ & 154 & $58.9(10.8)$ & $11.5^{* * *}$ & 485 (157) & $-191.4^{* * *}$ \\
\hline & & & IA8W & 151 & $59.4(10.9)$ & $11.7^{* *}$ & $479(154)$ & $-191.1^{* *}$ \\
\hline & & & Laser & 154 & $59.7(10.9)$ & 6.3 & $483(153)$ & -83.9 \\
\hline & \multirow{3}{*}[74]{} & \multirow{3}{*}{148} & IA4W & 154 & $58.9(10.8)$ & $10.4^{* * *}$ & $485(157)$ & $-200.4^{* * *}$ \\
\hline & & & IA8W & 151 & $59.4(10.9)$ & $10.5^{* * *}$ & $479(154)$ & $-190.1^{* * *}$ \\
\hline & & & Laser & 154 & $59.7(10.9)$ & 1.4 & $483(153)$ & -109.8 \\
\hline \multirow{9}{*}{ VIVID } & \multirow{3}{*}[70]{} & \multirow{3}{*}{52} & IA4W & 136 & $60.8(10.7)$ & $10.5^{* * *}$ & $502(144)$ & $-195.0^{* * *}$ \\
\hline & & & IA8W & 135 & $58.8(11.2)$ & $10.7^{* * *}$ & $518(147)$ & $-192.4^{* * *}$ \\
\hline & & & Laser & 132 & $60.8(10.6)$ & 1.2 & $540(152)$ & -66.2 \\
\hline & \multirow{3}{*}[70]{} & \multirow{3}{*}{100} & IA $4 \mathrm{~W}$ & 136 & $60.8(10.7)$ & $11.8^{* *}$ & $502(144)$ & $211.8^{* * *}$ \\
\hline & & & IA8W & 135 & $58.8(11.2)$ & $10.6^{* *}$ & $518(147)$ & $-195.8^{* * *}$ \\
\hline & & & Laser & 132 & $60.8(10.6)$ & 5.5 & $540(152)$ & -85.7 \\
\hline & \multirow{3}{*}[74]{} & \multirow{3}{*}{148} & IA4W & 136 & $60.8(10.7)$ & $10.3^{* * *}$ & $502(144)$ & $215.2^{* * *}$ \\
\hline & & & IA8W & 135 & $58.8(11.2)$ & $11.7^{* * *}$ & $518(147)$ & $-202.8^{* * *}$ \\
\hline & & & Laser & 132 & $60.8(10.6)$ & 1.6 & $540(152)$ & -122.6 \\
\hline \multirow{3}{*}{ Baker et al } & \multirow{3}{*}[80]{} & \multirow{3}{*}{104} & IA4W & 226 & $85.2(3.5)$ & $0.9(6.4)$ & $306(55)$ & $-48(65)$ \\
\hline & & & Laser & 240 & $85.2(3.8)$ & $0.1(6.3)$ & $314(52)$ & $-41(75)$ \\
\hline & & & Observation & 236 & $85.2(3.8)$ & $-0.4(6.4)$ & $314(64)$ & $-42(75)$ \\
\hline
\end{tabular}

Note. ${ }^{*} p<0.01$ vs. laser. ${ }^{* *} p<0.001$ vs. laser. ${ }^{* * *} p<0.0001$ vs. laser. Baker et al. measured central subfoveal thickness. w: weeks; BCVA: best-corrected visual acuity; EDTRS: Early Treatment of Diabetic Retinopathy Study; CRT: central retina thickness; IA4W: intravitreal injections of aflibercept every 4 weeks; IA8W: intravitreal injections of aflibercept every 8 weeks.

through anti-inflammatory properties [94] and VEGF inhibition [95]. Corticosteroids stabilize retinal capillaries and tend to reduce their permeability decreasing the leakage of plasma proteins into the interstitial tissue compartment $[8,9,96]$.

Although a single-dose preparation of preservative-free triamcinolone acetonide (Triesence ${ }^{\circledR}$; Alcon Laboratories, Inc., Fort Worth, TX, USA) has been approved by the FDA to enhance visualization of the vitreous during pars plana vitrectomy and to treat some posterior segment inflammatory diseases [97], it has not been approved for the treatment of DME. That is why triamcinolone acetonide would not be analyzed in this review.

\subsubsection{Dexamethasone Sustained-Release Implants.} Dexamethasone intravitreal (DEX) implant (0.7 mg) (Ozurdex, Allergan, Inc., Irvine, CA, USA) consists of micronized dexamethasone in a biodegradable copolymer of polylactic-co-glycolic acid which slowly releases steroids into the vitreous over a period of about 6 months $[98,99]$. In 2014, based on the results of the MEAD study [100], the FDA and most European countries approved Ozurdex for the treatment of DME.

The first prospective, randomized, and controlled trial evaluating the efficacy and safety of an intravitreal DEX in eyes with DME was published in 2010 [101]. Eyes with persistent DME ( $\geq 90$ days of duration) were randomly assigned to receive a DEX implant $(700 \mu \mathrm{g}$ or $350 \mu \mathrm{g})$ or observation. At month 3, the proportion of eyes achieving a BCVA improvement of $\geq 10$ letters was $33.3 \%, 21.1 \%$, and
$12.3 \%$ in the $700 \mu \mathrm{g} \mathrm{DEX}, 350 \mu \mathrm{g} \mathrm{DEX}$, and controls, respectively (700 $\mu \mathrm{g}$ DEX vs. controls, $p=0.007)$ [101].

Additionally, better anatomic outcomes were observed with the $700 \mu \mathrm{g}$ DEX than in the control group $(p=0.03)$. However, at month 6, there were no significant differences between groups [101]. According to the results of this clinical trial, $700 \mu \mathrm{g}$ DEX was an effective option for treating persistent DME, but its effects seemed to be time-limited [101].

The CHAMPLAIN was a prospective, multicenter, openlabel, and 26-week study that evaluated the efficacy and safety of a $700 \mu \mathrm{g}$ DEX for the treatment of DME in vitrectomized eyes [102]. At week 26, $700 \mu \mathrm{g}$ DEX significantly reduced the mean CMT $(p=0.004)$ and significantly improved the mean BCVA $(p=0.046)$ as compared to baseline. At week 8, the proportion of eyes that achieved a BCVA of $\geq 10$ letters were $30.4 \%$ [102]. The most commonly reported ocular adverse events were conjunctival hemorrhage, conjunctival hyperemia, elevation of IOP, and eye pain [102].

The PLACID study was a multicenter, prospective, randomized, controlled, double-masked, parallel-group, and 12 -month clinical trial that compared the $0.7 \mathrm{mg}$ DEX implant (Ozurdex ${ }^{\circledR}$ ) in combination with laser therapy vs. laser alone for the treatment of diffuse DME [103]. A total of 253 DME patients were randomly assigned to $0.7 \mathrm{mg}$ DEX + laser (at month 1) or sham implant + laser. Patients could receive up to 3 additional laser treatments and 1 additional DEX implant or sham treatment as needed [103]. The proportion of eyes with a BCVA improvement of $\geq 10$ letters was significantly greater at months 1 and 9 in the combination group than in the laser group $(p<0.001$ and 
$p=0.007$, respectively), although at month 12 such a difference was not significant [103]. Additionally, the area of vascular leakage was significantly decreased in the combination group as compared with the laser group $(p=0.041)$. Regarding safety, the incidence of elevation of IOP was significantly greater in the combination therapy group than in the laser group, but no eyes in the combination group required glaucoma surgery. Cataract-related side effects were more frequent in the combination group $(22.2 \%)$ than in the laser group (9.5\%), although there was no difference in the number of cataract surgeries between groups (4 eyes in the combination group and 5 eyes in the laser alone group) [103].

One of the most important studies assessing the effect of DEX treatment in DME patients was the MEAD [100]. The MEAD was two prospective, multicenter, randomized, masked, sham-controlled, three-year, and phase III clinical trials designed to evaluate the efficacy and safety of DEX implant 0.7 and $0.35 \mathrm{mg}$ in patients with DME [100]. At baseline, study patients were randomly assigned $(1: 1: 1)$ to receive DEX implant $0.7 \mathrm{mg}$, DEX implant $0.35 \mathrm{mg}$, or a sham procedure. The proportion of patients achieving a BCVA improvement of $\geq 15$ letters at year 3 (or at the last study visit) was significantly greater with both DEX implant $0.7 \mathrm{mg}(22.2 \%, p<0.001$ vs. sham $)$ and DEX implant $0.35 \mathrm{mg}$ (18.4\%, $p=0.018$ vs. sham). Interestingly, the treatment effect was really fast; in fact, significant differences in the proportion of eyes gaining $\geq 15$ letters were observed as early as day 21 . The mean CMT reduction was significantly greater with $0.7 \mathrm{mg}$ DEX $(-111.6 \pm 134.1 \mu \mathrm{m})$ and $0.35 \mathrm{mg}$ DEX $(-107.9 \pm 135.8 \mu \mathrm{m})$ than with sham $(-41.9 \pm 116.0 \mu \mathrm{m})$, $p<0.001$ each [100].

Among phakic eyes at baseline, the incidence of cataractrelated side effects was $67.9 \%, 64.1 \%$, and $20.4 \%$ in the $0.7 \mathrm{mg}$ DEX, $0.35 \mathrm{mg}$ DEX, and sham, respectively. The rate of cataract surgery was $59.2 \%, 52.3 \%$, and $7.2 \%$ in the $0.7 \mathrm{mg}$ DEX, $0.35 \mathrm{mg}$ DEX, and sham, respectively. An increase in IOP was observed in $27.7 \%, 24.8 \%$, and $3.7 \%$ of the eyes underwent $0.7 \mathrm{mg}$ DEX, $0.35 \mathrm{mg}$ DEX, and sham, respectively. Five (1.4\%) eyes in the 0.7 DEX, $3(0.9)$ in the 0.35 DEX, and $1(0.3 \%)$ sham required a glaucoma procedure (trabeculoplasty, iridotomy, iridectomy, or trabeculectomy) [100].

The BEVORDEX study was a phase II, prospective, multicenter, randomized, single-masked clinical trial that compared the 0.7 DEX implant Ozurdex vs. bevacizumab in patients with DME [104]. This study enrolled 88 eyes from 61 patients who were randomized to receive DEX (46 eyes) every 16 weeks or bevacizumab ( 42 eyes) every 4 weeks, both PRN. At month 12, the proportion of eyes having a BCVA improvement of $\geq 10$ letters was $41 \%(19 / 46)$ and $40 \%$ (17/ $42)$ in the DEX and bevacizumab groups, respectively $(p=0.83)$. The mean CMT reduction was significantly greater with DEX $(187 \mu \mathrm{m})$ than with bevacizumab $(122 \mu \mathrm{m})$, $p=0.015$. The mean number of intravitreal injections with DEX (2.7) was significantly lower than with bevacizumab (8.6) $[104]$

The eyes included in the BEVORDEX study continued in the trial for another year on the same treatment allocation, and $68(77 \%)$ of the 88 enrolled eyes completed the 24month trial [105]. The results of the BEVORDEX study at 2 years found that 43\% (20/46) DEX and 45\% (19/42) bevacizumab-treated eyes achieved a BCVA improvement of $\geq 10$ letters $(p=0.99)(105)$. At month 24 , there were significant differences between groups in the mean CMT reduction. Although, during the second year, the mean number of intravitreal injections with bevacizumab $(4.8 \pm 5.1)$ was greater than that of DEX $(2.2 \pm 1.2)$, the difference was less pronounced than during the first year $[104,105]$.

The efficacy and safety of DEX implant in DME eyes that did not adequately respond to three monthly intravitreal injections of anti-VEGF were evaluated in a prospective clinical trial [106]. The results of this study suggested that DEX significantly improved BCVA at month $2(p=0.0381)$ and significantly reduced CMT at months 1,2 , and 3 ( $p=0.0343, p=0.0288$, and $p=0.0370$, respectively). In the negative side, as compared with baseline, the IOP was significantly greater at months 1,2 , and $3(p=0.0003$, $p=0.0003$, and $p=0.0048$, respectively) [106].

The results of the BEVORDEX 12-month study [104] were confirmed by a single-center, randomized, and subjectmasked study conducted on eyes with persistent DME [107]. The results of this study found no differences in the mean change in BCVA between DEX $(+5.8 \pm 7.6$ letters $)$ and bevacizumab $(+5.6 \pm 6.1)(p=0.785)$. Nevertheless, the mean change in CMT was significantly greater with DEX $(-122 \pm 120 \mu \mathrm{m})$ than with bevacizumab $(-13 \pm 105)$ $(p<0.001)$. Similarly, in the BEVORDEX 12-month study (171), the number of injections was significantly greater with bevacizumab $(7.0 \pm 0.2)$ than with DEX $(2.7 \pm 0.5)$ $(p<0.001)$ [107].

A single-masked, randomized controlled study determined whether combined therapy with DEX + intravitreal bevacizumab $(1.25 \mathrm{mg})$ provides better outcomes than bevacizumab monotherapy in DME eyes [104]. At month 12, BCVA improvement was significant and equivalent in both groups (+5.4 and +4.9 in the combined and bevacizumab monotherapy groups, respectively, $p=0.75$ ). Nevertheless, the central subfield thickness reduction was significantly greater with the combined therapy than with bevacizumab alone (mean difference, $69 \mu \mathrm{m}, 95 \%$ confidence interval $=9-129 ; p=0.03)$ [108].

Data collected from the MEAD trials were pooled [109]. This post hoc analysis aimed at comparing the long-term effects of DEX (either $0.7 \mathrm{mg}$ or $0.35 \mathrm{mg}$ ) on the anatomic outcomes. Patients were randomized $(1: 1: 1)$ to intravitreal DEX implant $0.7 \mathrm{mg}$, DEX implant $0.35 \mathrm{mg}$, or a sham procedure in the study eye. Of the 1,048 randomized patients of the intend-to-treat population, 607 (57.9\%) patients completed all visits. At the end of the study follow-up, the mean CMT reduction was significantly greater with both 0.7 $\operatorname{DEX}(117 \mu \mathrm{m})$ and $0.35 \mathrm{mg} \operatorname{DEX}(127.8 \mu \mathrm{m})$ than with the sham $(62.1 \mu \mathrm{m})$, (both $p<0.001$ vs. sham) [109].

The UDBASA was a multicenter, prospective, and randomized study, conducted on patients with DME, designed to evaluate a single administration vs. a PRN administration of a DEX [110]. Patients were randomly 
assigned to two groups: In Group I, patients were treated according to the MEAD protocol (only one DEX during the 6 months of follow-up) [100]; in Group II, patients were treated on as-needed basis (once received the first DEX, patients visited every month and based on BCVA and CMT receive a customized PRN treatment regime) [110]. As compared to baseline, BCVA significantly improved, in both groups, at months 1 and 3, and started to decline in Group I at month 6. Although a difference of $0.11 \log$ MAR in BCVA between groups was observed (in favor of the PRN regime), it was not statistically significant. A statistically significant CMT reduction in both groups was observed up to month 2, but at that time Group I had begun to revert to pretreatment level. CMT reduction from baseline at months 4 and 5 was statistically significant in favor of the PRN regime $(p<0.05)$. The mean number of DEX in Group II was 1.6 vs. 1 in Group I [110]. The proportion of patients with an increased IOP requiring glaucoma medical therapy was $14 \%$ and $30 \%$ in Groups I and II, respectively $(p=0.13)$. The need of cataract surgery was similar in both groups $(48 \%$ and $40 \%$ in Groups I and II, respectively, $p=0.26$ [110].

A phase II prospective, randomized, and multicenter clinical trial compared, in patients with persistent DME, the effect of two treatment strategies: continued ranibizumab alone vs. continued ranibizumab plus DEX [111]. DME pseudophakic eyes with a BCVA score of between 24 and 78 letters and previously treated with anti-VEGF therapy (at least 3 anti-VEGF injections) were included in the study. There was a run-in period, where the patients received treatment with 3 intravitreal injections of ranibizumab. Those eyes that met the inclusion/exclusion criteria after the 12 -week run-in period were randomized $(1: 1)$ to receive either intravitreal ranibizumab + sham implant or intravitreal ranibizumab + DEX. BCVA improvement was similar in both groups (mean difference, 0.5 letters, $p=0.73$ ). The mean CMT reduction was significantly greater in those eyes treated with ranibizumab + DEX than in those who receive ranibizumab alone (mean difference, $52 \mu \mathrm{m} ; p<0.001$ ). The incidence of either increased IOP or initiation of glaucoma medical therapy was significantly higher in the ranibizumab + DEX (29\%) than in the ranibizumab alone treated eyes $(0 \%), p<0.001[111$.

Table 5 summarizes the main results of the DEX implant.

Besides the good efficacy and safety profile of DEX reported in the clinical trials [100-111], the efficacy and safety of Ozurdex ${ }^{\circledR}$ for the treatment of DME have been recently evaluated in clinical and real-life studies [113-120]. In summary, the results of these studies clearly indicated that Ozurdex ${ }^{\circledR}$ significantly improved the functional (visual acuity) and anatomic (retinal thickness) outcomes, not only in the midterm $[113,114,119,120]$ but also in the long term [115-118], in both naïve and previously treated DME patients, but naïve eyes consistently fared better [113-118, 120].

Malclès et al. [116], in a retrospective and bicentric study, evaluated the efficacy and safety of DEX in DME patients in real-life practice over a period of 3 years. The results of this study found a significant improvement in BCVA (9.5 letters at month $36, p=0.023$ ), with $25.4 \%$ of eyes achieving at least a 15-letter improvement at month 36. Additionally, there was a significant decrease in CMT at month 36.

A retrospective and multicenter study assessed the efficacy and safety of repeated DEX, over a 24-month followup period, in DME eyes, either naïve or refractory to antiVEGF, in a real setting [118]. The results of this study showed that although both, naive and refractory eyes, improved significantly in vision after 24 months $(p<0.001)$, BCVA improvement was significantly greater in the naïve eyes that in the refractory ones $(p<0.01$.) A statically significant CMT reduction was observed in both groups [118].

When treating DME patients, one important question is to know when to change a treatment strategy and what treatment to choose. Despite that the anti-VEGF therapy has been chosen as first-line therapy [6], many eyes do not adequately respond to them. A post hoc analysis of the DRCR.net Protocol I revealed that $40 \%$ of eyes achieved a BCVA improvement $<5$ letters at week 12 [59]. Additionally, eyes with a poor response to ranibizumab (those gaining $<5$ letters after three intravitreal ranibizumab injections administered monthly) usually do not improve further with continuing in ranibizumab treatment [59].

Moreover, extending the dose to 24 weeks did not provide better functional or anatomic outcomes [78].

However, the question of whether patients who do not adequately respond to anti-VEGF could benefit from an early change to another therapy has not been fully elucidated.

A retrospective, multicenter, and case-control study, conducted in a real setting, compared the effect of continuing with an anti-VEGF therapy or switching to a DEX in eyes with refractory DME after three initial anti-VEGF injections [121]. One hundred and ten (72 eyes in the antiVEGF and 38 in the DEX groups) were included in the study. The mean change in BCVA was significantly greater in the $\operatorname{DEX}(+6.1 \pm 10.6$ letters $)$ than in the anti-VEGF $(+0.4 \pm 10.8$ letters) group, $p=0.004$. The mean CMT reduction was significantly greater in the DEX $(-92.8 \pm 173.6 \mu \mathrm{m})$ than in the anti-VEGF $(+18.3 \pm 145.9 \mu \mathrm{m})$ group, $p<0.001$. At month 12 , the probability of achieving a BCVA improvement of $\geq 10$ letters was significantly greater in the DEX than in the anti-VEGF group (odds ratio, 3.71; 95\% confidence interval, 1.19-11.61; $p=0.024)$ [121].

The effect of early (DME eyes receiving 3 or fewer antiVEGF injections before switch) vs. late switch (DME eyes receiving 6 or more anti-VEGF injections before switch) on BCVA and CMT was compared in a retrospective study [122]. As compared to baseline, BCVA significantly improved in the early-switch group at month $24(p=0.043)$ but did not in the late-switch group $(p=0.8602)$. The CMT was significantly reduced in both early- and late-switch groups ( $p=0.0002$ and $p=0.0038$, respectively). Nevertheless, the proportion of eyes obtaining a CMT reduction $\geq 10 \%$ was significantly greater in the early-switch group than in the late-switch one $(71.0 \%$ vs. $47.4 \%, p=0.0498)$. There was no difference in the incidence of IOP increase between both groups [122].

These results were partially confirmed by a retrospective study, which found that, at month 6 , the change in central 
TABLE 5: Overview of the functional and anatomic results of dexamethasone intravitreal (DEX) and fluocinolone intravitreal implants.

\begin{tabular}{|c|c|c|c|c|c|c|c|c|}
\hline \multirow{2}{*}{ Study } & \multirow{2}{*}{ Ref. } & \multirow{2}{*}{ Duration (m) } & \multirow{2}{*}{ Regimen } & \multirow{2}{*}{$N$ (eyes) } & \multicolumn{2}{|c|}{ BCVA (ETDRS letters) } & \multicolumn{2}{|c|}{ CRT $(\mu \mathrm{m})$} \\
\hline & & & & & Baseline & Change & Baseline & Change \\
\hline \multirow{3}{*}{ MEAD } & \multirow{3}{*}[100]{} & \multirow{3}{*}{36} & DEX $0.35 \mathrm{mg}$ & 351 & $56.1(9.9)$ & $18.4 \%^{1 * * *}$ & $463.0(157.1)$ & $-107.9(135.8)^{* * *}$ \\
\hline & & & DEX $0.7 \mathrm{mg}$ & 347 & $55.5(9.7)$ & $22.2 \%^{1 * *}$ & $466.8(159.5)$ & $-111.6(134.1)^{* * *}$ \\
\hline & & & Sham & 350 & $56.9(8.7)$ & $12.0 \%^{1}$ & $460.9(132.6)$ & $-41.9(116.0)$ \\
\hline \multirow{4}{*}{ BEBORDEX } & \multirow{2}{*}[104]{} & \multirow{2}{*}{12} & DEX $0.7 \mathrm{mg}$ & 46 & $55.5(12.5)$ & $7.9(11.6)$ & $474.3 \pm 95.9$ & $-179.0(88.8)^{* *}$ \\
\hline & & & Beva $0.5 \mathrm{mg}$ & 42 & $56.3(11.9)$ & $7.5(11.0)$ & $503 \pm 140.9$ & $-93.0(131.6)$ \\
\hline & \multirow{2}{*}[105]{$^{\dagger}$} & \multirow[b]{2}{*}{24} & DEX $0.7 \mathrm{mg}$ & 46 & $55.5(12.5)$ & $6.9(2.7$ to 11.1$)$ & $474.3 \pm 95.9$ & N.A. \\
\hline & & & Beva $0.5 \mathrm{mg}$ & 42 & $56.3(11.9)$ & $9.6(6.9$ to 12.3$)$ & $503 \pm 140.9$ & N.A. \\
\hline \multirow{2}{*}{ Shah et al } & \multirow{2}{*}[107]{} & \multirow[t]{2}{*}{7} & DEX $0.7 \mathrm{mg}$ & 27 & $59(12)$ & $5.8(7.6)$ & $458(100)$ & $-122(95)^{* * *}$ \\
\hline & & & Beva $0.5 \mathrm{mg}$ & 23 & $59(13)$ & $5.6(6.1)$ & $485(122)$ & $-14(141)$ \\
\hline \multirow{2}{*}{ Maturi et al } & \multirow{2}{*}[108]{} & \multirow{2}{*}{6} & DEX + Rani & 65 & $63(12)$ & $2.7(9.8)$ & $375(97)$ & $-110(86)^{* * *}$ \\
\hline & & & Sham + Rani & 64 & $63(13)$ & $3.0(7.1)$ & $3968122)$ & $-62(97)$ \\
\hline \multirow{3}{*}{ FAME } & \multirow{3}{*}[132]{} & \multirow{3}{*}{24} & IFSR $0.2 \mu \mathrm{g}$ & 375 & $53.3(12.7)$ & $p=0.019$ & $460.8(160.0)$ & $p \leq 0.003$ \\
\hline & & & IFSR $0.5 \mu \mathrm{g}$ & 393 & $52.9(12.2)$ & $p=0.015$ & $485.1(173.8)$ & $p \leq 0.003$ \\
\hline & & & Sham & 185 & $54.7(11.3)$ & - & $451.3(152.0)$ & - \\
\hline \multirow{2}{*}{ FAME $^{\ddagger}$} & \multirow{2}{*}[133]{} & \multirow{2}{*}{36} & IFSR $0.2 \mu \mathrm{g}$ & 165 & 54.7 (11.7) & 2.4 & $466.6(152.9)$ & $-173.1^{*}$ \\
\hline & & & Sham & 72 & 557. (11.5) & 2.3 & $435.0(149.1)$ & -115.6 \\
\hline \multirow{2}{*}{$\mathrm{FAME}^{\ddagger \ddagger}$} & \multirow{2}{*}[133]{} & \multirow{2}{*}{36} & IFSR $0.2 \mu \mathrm{g}$ & 209 & $52.2(13.4)$ & $7.6^{* *}$ & $456.2(165.9)$ & $\begin{array}{l}-186.8 \\
\end{array}$ \\
\hline & & & Sham & 112 & $54.0(11.5)$ & 1.8 & $461.8(153.5)$ & -160.0 \\
\hline
\end{tabular}

Note. ${ }^{*} p<0.05$ vs. comparator/sham. ${ }^{* *} p<0.01$ vs. comparator/sham. ${ }^{* * *} p<0.001$ vs. comparator/sham. ${ }^{* * * *} p<0.0001$ vs. comparator/sham. ${ }^{1}$ Proportion of patients with a $\geq 15$-letter improvement in best-corrected visual acuity (BCVA) from baseline at the year 3 . ${ }^{\dagger}$ For those eyes that were pseudophakic at baseline, the mean improvement in BCVA was 8.9 letters (95\% confidence interval (CI), 2.0-13.4) for those treated with the dexamethasone (DEX) implant and 7.7 letters $(95 \% \mathrm{CI}, 3.03-14.8)$ for those treated with bevacizumab; $p=0.77$. For the eyes that were phakic at baseline, the mean improvement in BCVA was 5.8 letters (95\% CI, 0.07-11.5) for those treated with the DEX implant and 10.2 letters (95\% CI, 7.17-13.3) for those treated with bevacizumab; $p=0.19$. The specific data regarding central retinal thickness of BEVORDEX study at 24 months are not available from the literature [105] and hence are not listed in this table. Shah et al. [107] measured central subfoveal thickness. Maturi et al. [108] measured central subfoveal thickness. FAME: The specific data regarding BCVA and central retinal thickness are not available from the literature [112] and hence are not listed in this table. The $p$ value corresponded to the difference between intravitreal fluocinolone sustained-release (IFSR) and sham. ${ }^{*}$ Nonchronic diabetic macular edema (DME) (<3 years). ${ }^{\ddagger \ddagger} \mathrm{Chronic}$ DME ( $\geq 3$ years). m: months; BCVA: best-corrected visual acuity; EDTRS: Early Treatment of Diabetic Retinopathy Study; CRT: central retina thickness; DEX: dexamethasone implant; Beva: bevacizumab; IFSR: intravitreal fluocinolone sustained-release.

retinal thickness was significantly better in the early-switch group than in the late-switch group [123].

Finally, the results of a retrospective study published recently suggested that, in DME patients who did not adequately respond to 3 monthly intravitreal anti-VEGF injections, switching to dexamethasone implant provided better functional outcomes than those that received $>3$ antiVEGF injections [124].

(1) DEX Implant in Cataract Surgery. Cataract surgery is a common and safe procedure but can be associated with vision-threatening complications in the diabetic population, such as diabetic macular edema, postoperative macular edema, diabetic retinopathy progression, and posterior capsular opacification $[125,126]$.

Different hypotheses about the mechanisms involved in the pathogenesis of cataract in diabetic patients have been proposed, including polyol pathway, osmotic and oxidative stress, or autoimmunity [127]. Besides preoperative counselling, which is crucial for diabetic patients, other aspects such as glycemic control, evidence of ocular inflammation, history of preexisting proliferative diabetic retinopathy, and/or macular edema should be taken into consideration before cataract surgery in diabetic patients [125-127].

Since preexisting DME can increase the risk of macular edema progression by $20 \%-50 \%$, an appropriate therapeutic management of DME is recommended perioperatively [128]. Surgical inflammation associated with cataract surgery may be responsible for poor functional outcomes in DME patients [125-127]. Moreover, diabetic patients have a substantial risk of developing DME after cataract surgery and particularly in the 3- to 6-month postoperative period [125]. Therefore, the perioperative administration of a DEX implant in diabetic patients undergoing cataract surgery might be beneficial.

We have evidence suggesting that, in diabetic patients, the intraoperative use of a DEX implant in combination with phacoemulsification and IOL implantation could provide good functional and anatomic outcomes [129-131].

A prospective study, conducted on 19 eyes of patients with type 2 diabetes mellitus with DME, who underwent cataract surgery, found that intraoperative DEX implant effectively prevented DME worsening after phacoemulsification [129]. Similarly, Furino et al. [130] reported that intraoperative intravitreal DEX provided good functional and anatomic clinical outcomes in DME patients who underwent cataract surgery and these positive effects last for at least 3 months. Moreover, the results of a prospective, singlearm, and open-label study suggested that prophylactic use of intraoperative DEX resulted in excellent anatomic outcomes in DME undergoing cataract surgery [131]. 
A retrospective and comparative cohort study published recently compared anatomical and functional outcomes of combined phacoemulsification and dexamethasone intravitreal implant with standard phacoemulsification in patients with nonproliferative diabetic retinopathy, ME, and cataract [132]. The results of this study found that, in those patients who underwent combined phacoemulsification and DEX implant, there was a significant and maintained increase in BCVA and a significant decrease in central subfoveal thickness throughout the study. The IOP significantly increased during the follow-up in the combined phacoemulsification and DEX implant group, although it remained within the normal range. According to the results of this study, in diabetic patients with DME, combined treatment with phacoemulsification and DEX implant provided better functional and anatomic outcomes than standard phacoemulsification [132].

3.2.2. Fluocinolone Sustained-Release Implants. The efficacy and safety of two intravitreal fluocinolone sustained-release (IFSR) devices $0.2 \mu \mathrm{g} /$ day (low-dose) or $0.5 \mu \mathrm{g} /$ day (highdose) were assessed in DME patients. Two parallel, prospective, randomized, sham injection-controlled, doublemasked, multicenter clinical trials (FAME trials) were conducted on patients with persistent DME who received at least 1 macular laser treatment. Study subjects were randomized $(2.2: 1)$ to low-dose implant, high-dose implant, or sham implant, respectively [112]. At month 24, the proportion of patients achieving a BCVA improvement of $\geq 15$ letters was significantly greater in both low-and high-dose groups than in the sham group ( $p=0.002$ each). Mean BCVA improvement was $4.4,5.4$, and 1.7 in the low-dose, high-dose, and sham groups, respectively (low-dose vs. sham, $p=0.02$, and highdose vs. sham $p=0.016$ ). As compared with sham, CMT reduction was significantly greater in both low and high dose at all the time points measured. The need of glaucoma surgery was $3.7 \%, 7.6 \%$, and $0.5 \%$ in the low-dose, high-dose, and sham groups, respectively [112] (Table 5).

In a subgroup analysis of the FAME trials, the efficacy and safety of an IFSR $0.2 \mu \mathrm{g} /$ day or sham in eyes with chronic (duration of diagnosis, $\geq 3$ years) and nonchronic (duration of diagnosis, $<3$ years) DME were assessed [133]. At month 36 , proportion of patients gaining $\geq 15$ letters in BCVA was significantly greater in chronic DME patients (IFSR $0.2 \mu \mathrm{g} /$ day, $34.0 \%$ vs. sham, $13.4 \%$; $p<0.001)$, but not in patients with nonchronic DME (IFSR $0.2 \mu \mathrm{g} /$ day, $22.3 \%$ vs. sham, $27.8 \% ; p=0.275$ ). The greater functional response observed in the chronic DME eyes was not associated with baseline ocular characteristics, changes in anatomic features, or differences in retreatment or ancillary therapies. Duration of DME did not influence the incidence of adverse events [133].

A post hoc analysis of the FAME trials evaluated the treatment outcomes in phakic eyes who received IFSR $0.2 \mu \mathrm{g} /$ day implant [134]. At month 36 , the proportion of eyes achieving a BCVA improvement of $\geq 15$ letters was slightly higher in the eyes that had cataract surgery after (35.1\%) than in the eyes that had cataract surgery before (29.3\%). Additionally, as compared with nonchronic DME
(27.5\%), a greater proportion of eyes with chronic DME (42.3\%) achieved a BCVA improvement of $\geq 15$ letters [134].

Elevated IOP was more common in IFSR than in sham control-treated patients. There was no evidence that either glaucoma surgery, laser, or topical medication significantly impacted visual outcomes. None of the previously treated eyes with steroids that receive treatment with an IFSR $0.2 \mu \mathrm{g} /$ day implant required IOP-lowering surgery [135].

The long-term efficacy and safety of an IFSR were assessed in a prospective, randomized, evaluator-masked, controlled, and multicenter study. Study patients were randomized $(2: 1)$ to receive either $0.59 \mathrm{mg}$ IFSR or standard of care (additional laser or observation) [136]. At 2 years, the proportion of eyes achieving a BCVA improvement $\geq 3$ lines were $31.8 \%$ and $9.3 \%$ in the IFSR and standard-of-care groups, respectively, $p=0.0016$. However, at 3 years, such difference was not statistically significant $(p=0.1566)$. The proportion of eyes with no evidence of retinal thickening was significantly higher in the standard-of-care group than in the IFSR at month 6 , and years 1 and $2(p<0.0001$, $p<0.0001$, and $p=0.016$, respectively), but not at year 3 $(p=0.861)$. An IOP $\geq 30 \mathrm{mmHg}$ (at any time point visit) was observed in $61.4 \%$ and $5.8 \%$ of the IFSR and standard-ofcare groups, respectively. $33.8 \%$ of the eyes treated with IFSR required surgery for ocular hypertension by 4 years [136].

The approved dose for the fluocinolone acetonide implant (Iluvien ${ }^{\circledast}$; Alimera Sciences, Inc., Alpharetta, GA, USA) was $0.19 \mathrm{mg}$. In theory, it is delivered over 36 months at a rate of $0.2 \mu \mathrm{g} / \mathrm{day}$ [137]. However, pharmacokinetic studies reported that IFSR provides sustained delivery for approximately one year [138].

To date, there have been numerous papers looking at the real-world efficacy and safety profile of the IFSR implant at years 1 and 2 and data are emerging for 3 years. The results of these studies are in the same general direction, indicating that, on average, IFSR implant significantly improved BCVA and reduced CMT in DME patients [139-148].

The ILUVIEN Implant for chronic DiabEtic MAcuLar edema (Retro-IDEAL) was a retrospective study designed for assessing the efficacy and safety of an IFSR $(0.19 \mathrm{mg})$ in patients with chronic DME in Germany [143]. The results at month 30 found that, from baseline, BCVA significantly improved $(p<0.05)$. Additionally, CMT was significantly reduced at year $3(p<0.001)$ [144].

\subsection{Emerging Therapies}

3.3.1. Designed Ankyrin Repeat Protein (DARPin). Designed ankyrin repeat protein (DARPin), which derives from natural ankyrin repeats, is a small, single-domain protein that can selectively bind to a target protein with high affinity and specificity $[149,150]$.

Besides their high selectivity and affinity, DARPin molecules also display remarkable stability that confer some advantages over currently available antibodies or antibody fragments as potential therapeutics. In addition, DARPin molecules can be specifically designed to modulate local or systemic pharmacokinetics $[150,151]$. 
Abicipar pegol (AGN-150998, MP0112, abicipar; Allergan plc/Molecular Partners) is an antagonist of VEGFA characterized by small size, high potency, and long intravitreal half-life $[15,152]$. A phase I/II, open-label, multicenter dose-escalation trial, evaluating the safety and bioactivity of abicipar pegol in DME patients, found that there were prolonged edema reduction and improvement in vision [153]. Similarly, the results from a phase II study showed that abicipar pegol, injected every 8 or 12 weeks in patients affected by DME, offered the functional and anatomical effects with less frequent injections compared with ranibizumab over a 28-week period [154].

3.3.2. Brolucizumab. Brolucizumab (Beovu $\left.{ }^{\circledR}\right)$ is a lowmolecular-weight, single-chain antibody fragment antiVEGF developed by Novartis (Basel, Switzerland) for the treatment of neovascular age-related macular degeneration, DME, and macular edema secondary to retinal vein occlusion [155].

A $6 \mathrm{mg}$ dose of brolucizumab delivers a molar dose which is about 11 and 22 times higher than aflibercept $2 \mathrm{mg}$ and ranibizumab $0.5 \mathrm{mg}$, respectively.

This drug has been already compared to aflibercept in patients with neovascular age-related macular degeneration $[156,157]$. The HAWK and HARRIER, two similarly designed phase III clinical trials conducted on patients with neovascular age-related macular degeneration, showed that brolucizumab was not inferior to aflibercept in visual function at week 48, although anatomic outcomes favored brolucizumab over aflibercept [157]. Regarding safety, it should be mentioned that intraocular inflammation was identified in $50(4.6 \%)$ of the brolucizumab-treated patients. Of those, 36 subjects (3.3\%) had concomitant retinal vasculitis. Of the 36 subjects with intraocular inflammation and vasculitis, 23 subjects $(2.1 \%)$ had concomitant vascular occlusion [157]. The American Society of Retina Specialists (ASRS) conducted a postapproval analysis of brolucizumabassociated retinal vasculitis after cases [158]. Retinal vasculitis was reported in 26 eyes of 25 patients after treatment with brolucizumab (of which $85 \%$ were designated as occlusive). Twelve eyes (46\%) had a greater than 3-line decrease in VA at the final follow-up, and 12 eyes (46\%) had a final VA of 20/200 or worse [158]. Additionally, a retrospective case series found that retinal vasculitis and intraocular inflammation after intravitreal injection of brolucizumab were characterized by variable occlusion of large or small retinal arteries, or both, and perivenular abnormalities [159].

However, up to now, there are no published data in DME patients. A 2-year, randomized, double-masked, multicenter, active controlled study is ongoing to compare the efficacy and safety of brolucizumab $3 \mathrm{mg}$ and brolucizumab $6 \mathrm{mg}$ vs. aflibercept $2 \mathrm{mg}$. The estimated study completion date will be October 2021, and its results will be available in the next few years [160].

Additionally, the ongoing prospective, randomized, phase III clinical study in DME, KITE, aims to confirm the noninferiority of brolucizumab $6 \mathrm{mg}$ compared to aflibercept $2 \mathrm{mg}$ on a functional and morphological level as well as durability effect over 2 years [161]. However, its results have not been published yet.

3.3.3. Angiopoietin Combination Drugs. Angiopoietins are a family of growth factors that bind to endothelial receptor tyrosine kinases [162]. Angiopoietin-2 is considered a key factor in DME pathogenesis (see Figure 1) [9, 152].

As far as we know, there are some ongoing clinical trials targeting angiopoietins in DME patients $[11,152]$. The BOULEVARD trial was a prospective, randomized, and multicenter clinical trial that compared the efficacy and safety and efficacy of antibody targeting angiopoietin-2 and VEGF-A vs. ranibizumab in patients with DME [163]. The angiopoietin-2 inhibitor demonstrated statistically superior VA gains than ranibizumab at week 24 in treatment-naïve patients [163].

\section{Conclusions}

Many different options are currently available for treating DME. Although laser photocoagulation was considered the gold standard treatment for DME for several years, it is not longer.

Nowadays, intravitreal corticosteroids and anti-VEGF have become first line for treating DME. The anti-VEGF of choice might depend on baseline BCVA. While aflibercept may be the drug of choice in patients with low baseline BCVA (approximately 20/50 or worse); the three antiVEGFs (bevacizumab, ranibizumab, and aflibercept) provide similar functional outcomes in patients with higher baseline BCVA (approximately 20/32 to 20/40).

Since the identification of the role of inflammation, corticosteroids have taken an active role in the treatment of DME. Intravitreal corticosteroids represent a valuable option for treating DME. However, they are usually seen as a second choice, particularly in those eyes that have an insufficient response to anti-VEGF. Emerging evidence suggests that, in eyes that did not adequately respond to 3 anti-VEGF injections, switching to a DEX implant provides better functional outcomes than in those who received $>3$ anti-VEGF injections. This finding brings to the table the convenience of not extending anti-VEGF further in those eyes that exhibited an insufficient therapeutic response after three doses.

Since there seems to be a relationship between antiVEGF and vascular disorders, especially in elderly people, intravitreal corticosteroids would be the treatment of choice in patients at risk of suffering cardiovascular and/or cerebrovascular events. Another group of patients who could potentially benefit from corticosteroids as first-line therapy are those unwilling to follow the anti-VEGF treatment regime (monthly injections and/or monthly visits) during the first 6 months. At the time of choosing a corticosteroid, dexamethasone should be used first, reserving the use of fluocinolone for those DME cases that did not adequately respond to other treatments. Intravitreal triamcinolone is associated with a greater IOP increase and higher incidence of cataract, and its use should be reserved in those patients who cannot obtain the approved agents for this indication. 
DARPins against VEGF have been demonstrated to inhibit specifically angiogenesis in both in vitro and in vivo studies. These treatments have shown to be as effective as anti-VEGF, but with fewer doses, which may reduce the risk of serious adverse events, for example, endophthalmitis.

New therapies will probably come in the next years for treating DME. Also, novel drug delivery systems using nanotechnology, sustained-release delivery implants, and stem cell therapy are on the horizon.

\section{Data Availability}

No data were used to support the findings of this study.

\section{Disclosure}

Allergan did not participate in either data analysis or redaction of the manuscript.

\section{Conflicts of Interest}

Dr. Claudio Furino has received a grant from Allergan during the conduct of the study. Dr. Francesco Boscia, Dr. Michele Reibaldi, and Dr. Giovanni Alessio declare that they have no conflicts of interest.

\section{Authors' Contributions}

All authors met the ICMJE authorship criteria. All authors made substantial contributions to conception, design, analysis, and interpretation of data, contributed to the writing of the article, provided critical revision of the manuscript, and approved the final version.

\section{Acknowledgments}

Medical writing and Editorial assistant services have been provided by Ciencia y Deporte S.L. and covered by a Grant from Allergan. Support for this assistance was funded by Allergan, an AbbVie company. Logistic for writing services has been provided by Allergan, an AbbVie company.

\section{References}

[1] N. H. Cho, J. E. Shaw, S. Karuranga et al., "IDF Diabetes Atlas: global estimates of diabetes prevalence for 2017 and projections for 2045," Diabetes Research and Clinical Practice, vol. 138, pp. 271-281, 2018.

[2] R. R. A. Bourne, J. B. Jonas, A. M. Bron et al., "Prevalence and causes of vision loss in high-income countries and in Eastern and Central Europe in 2015: magnitude, temporal trends and projections," British Journal of Ophthalmology, vol. 102, no. 5, pp. 575-585, 2018.

[3] J. Q. Li, T. Welchowski, M. Schmid et al., "Prevalence, incidence and future projection of diabetic eye disease in Europe: a systematic review and meta-analysis," European Journal of Epidemiology, vol. 35, no. 1, p. 11, 2019.

[4] I. Kocur and S. Resnikoff, "Visual impairment and blindness in Europe and their prevention," British Journal of Ophthalmology, vol. 86, no. 7, pp. 716-722, 2002.

[5] J. Cunha-Vaz and G. Coscas, "Diagnosis of macular edema," Ophthalmologica, vol. 224, no. 1, pp. 2-7, 2010.
[6] U. Schmidt-Erfurth, J. Garcia-Arumi, F. Bandello et al., "Guidelines for the management of diabetic macular edema by the European society of retina Specialists (EURETINA)," Ophthalmologica, vol. 237, no. 4, pp. 185-222, 2017.

[7] R. A. Cantrell, F. Lum, Y. Chia et al., "Treatment patterns for diabetic macular edema: an intelligent Research in sight (IRIS) registry analysis," Ophthalmology, vol. 127, no. 3, pp. 427-429, 2020.

[8] A. Daruich, A. Matet, A. Moulin et al., "Mechanisms of macular edema: beyond the surface," Progress in Retinal and Eye Research, vol. 63, pp. 20-68, 2018.

[9] P. Romero-Aroca, M. Baget-Bernaldiz, A. Pareja-Rios, M. Lopez-Galvez, R. Navarro-Gil, and R. Verges, "Diabetic macular edema pathophysiology: vasogenic versus inflammatory," Journal of Diabetes Research, vol. 2016, p. 1, 2016.

[10] J. Tang and T. S. Kern, "Inflammation in diabetic retinopathy," Progress in Retinal and Eye Research, vol. 30, no. 5, pp. 343-358, 2011.

[11] A. I. Arroba and Á. M. Valverde, "Modulation of microglia in the retina: new insights into diabetic retinopathy," Acta Diabetologica, vol. 54, no. 6, pp. 527-533, 2017.

[12] The Diabetic Retinopathy Study Research Group, "Preliminary report on effects of photocoagulation therapy," American Journal of Ophthalmology, vol. 81, pp. 383-396, 1976.

[13] The Early Treatment Diabetic Retinopathy Study Research Group, "Photocoagulation for diabetic macular edema: early treatment diabetic retinopathy study report no. 4," International Ophthalmology Clinics, vol. 27, pp. 265-272, 1987.

[14] D. J. Browning, M. W. Stewart, and C. Lee, "Diabetic macular edema: evidence-based management," Indian Journal of Ophthalmology, vol. 66, no. 12, pp. 1736-1750, 2018.

[15] E. A. Urias, G. A. Urias, F. Monickaraj, P. McGuire, and A. Das, "Novel therapeutic targets in diabetic macular edema: beyond VEGF," Vision Research, vol. 139, pp. 221227, 2017.

[16] E. S. Gragoudas, A. P. Adamis, E. T. Cunningham Jr., M. Feinsod, and D. R. Guyer, "Pegaptanib for neovascular age-related macular degeneration," New England Journal of Medicine, vol. 351, no. 27, pp. 2805-2816, 2004.

[17] E. T. Cunningham Jr., A. P. Adamis, M. Altaweel et al., "Macugen Diabetic Retinopathy Study Group. A phase II randomized double-masked trial of pegaptanib, an antivascular endothelial growth factor aptamer, for diabetic macular edema," Ophthalmology, vol. 112, no. 10, pp. 1747-1757, 2005.

[18] M. B. Sultan, D. Zhou, J. Loftus, T. Dombi, and K. S. Ice, “A phase $2 / 3$, multicenter, randomized, double-masked, 2-year trial of pegaptanib sodium for the treatment of diabetic macular edema," Ophthalmology, vol. 118, no. 6, pp. 1107-1118, 2011.

[19] T. Ishibashi, M. Yuzawa, N. Yoshimura et al., "Japan phase 3 study of pegaptanib sodium in patients with diabetic macular edema," Nippon Ganka Gakkai Zasshi, vol. 118, no. 9, pp. 773-782, 2014.

[20] G. Querques, A. V. Bux, D. Martinelli, C. Iaculli, and N. D. Noci, "Intravitreal pegaptanib sodium (Macugen) for diabetic macular oedema," Acta Ophthalmologica, vol. 87, no. 6, pp. 623-630, 2009.

[21] M. Rinaldi, F. Chiosi, R. dell'Omo et al., "Intravitreal pegaptanib sodium (Macugen) for treatment of diabetic macular oedema: a morphologic and functional study," British Journal of Clinical Pharmacology, vol. 74, no. 6, pp. 940-946, 2012. 
[22] S. Sivaprasad, R. Browning, and C. Starita, "An open-label, one-year, noncomparative study to evaluate the safety and tolerability of intravitreal pegaptanib sodium in patients with diabetic macular edema," Clinical Ophthalmology, vol. 8, pp. 1565-1571, 2014.

[23] J. C. Cilley, K. Barfi, A. B. Benson 3rd., and M. F. Mulcahy, "Bevacizumab in the treatment of colorectal cancer," Expert Opinion on Biological Therapy, vol. 7, no. 5, pp. 739-749, 2007.

[24] C. Haritoglou, D. Kook, A. Neubauer et al., "Intravitreal bevacizumab (Avastin) therapy for persistent diffuse diabetic macular edema," Retina, vol. 26, no. 9, pp. 999-1005, 2006.

[25] J. F. Arevalo, J. Fromow-Guerra, H. Quiroz-Mercado et al., "Primary intravitreal bevacizumab (Avastin) for diabetic macular edema: results from the Pan-American Collaborative Retina Study Group at 6-month follow-up," Ophthalmology, vol. 114, no. 4, pp. 743-750, 2007.

[26] J. F. Arevalo, J. G. Sanchez, L. Wu et al., "Primary intravitreal bevacizumab for diffuse diabetic macular edema: the PanAmerican Collaborative Retina Study Group at 24 months," Ophthalmology, vol. 116, no. 8, pp. 1488-e1, 2009.

[27] A. Yanyali, B. Aytug, F. Horozoglu, and A. F. Nohutcu, "Bevacizumab (Avastin) for diabetic macular edema in previously vitrectomized eyes," American Journal of Ophthalmology, vol. 144, no. 1, pp. 124-126, 2007.

[28] L. Wu, M. A. Martínez-Castellanos, H. Quiroz-Mercado et al., "Pan American collaborative retina group (PACORES). Twelve-month safety of intravitreal injections of bevacizumab (avastin): results of the Pan-American collaborative retina study group (PACORES)," Graefe's Archive for Clinical and Experimental Opthomology, vol. 246, no. 1, pp. 81-87, 2008.

[29] Diabetic Retinopathy Clinical Research Network, I. U. Scott, I. U. Scott, and A. R. Edwards, "A phase II randomized clinical trial of intravitreal bevacizumab for diabetic macular edema," Ophthalmology, vol. 114, no. 10, pp. 1860-1867, 2007.

[30] M. Michaelides, A. Kaines, R. D. Hamilton et al., "A prospective randomized trial of intravitreal bevacizumab or laser therapy in the management of diabetic macular edema (BOLT study)," Ophthalmology, vol. 117, no. 6, pp. 1078-1086, 2010.

[31] R. Rajendram, S. Fraser-Bell, A. Kaines et al., "A 2-year prospective randomized controlled trial of intravitreal bevacizumab or laser therapy (BOLT) in the management of diabetic macular edema: 24-month data: report 3," Ophthalmology, vol. 130, no. 8, pp. 972-979, 2012.

[32] A. B. Nepomuceno, E. Takaki, F. P. Paes de Almeida et al., "A prospective randomized trial of intravitreal bevacizumab versus ranibizumab for the management of diabetic macular edema," American Journal of Ophthalmology, vol. 156, no. 3, pp. 502-510, 2013.

[33] K. Kriechbaum, S. Prager, S. Prager et al., "Intravitreal bevacizumab (Avastin) versus triamcinolone (Volon A) for treatment of diabetic macular edema: one-year results," Eye, vol. 28, no. 1, pp. 9-16, 2014.

[34] S. Sonoda, T. Sakamoto, T. Yamashita et al., "Effect of intravitreal triamcinolone acetonide or bevacizumab on choroidal thickness in eyes with diabetic macular edema," Investigative Opthalmology \& Visual Science, vol. 55, no. 6, pp. 3979-3985, 2014.

[35] Diabetic Retinopathy Clinical Research Network, J. A. Wells, J. A. Wells, and A. R. Glassman, "Aflibercept, bevacizumab, or ranibizumab for diabetic macular edema," The New
England Journal of Medicine, vol. 372, no. 13, pp. 1193-1203, 2015.

[36] J. A. Wells, A. R. Glassman, A. R. Ayala et al., "Diabetic retinopathy clinical Research Network. Aflibercept, bevacizumab, or ranibizumab for diabetic macular edema: twoyear results from a comparative effectiveness randomized clinical trial," Ophthalmology, vol. 123, no. 6, pp. 1351-1359, 2016.

[37] V. Ranpura, S. Hapani, J. Chuang, and S. Wu, "Risk of cardiac ischemia and arterial thromboembolic events with the angiogenesis inhibitor bevacizumab in cancer patients: a meta-analysis of randomized controlled trials," Ophthalmology, vol. 49, no. 3, pp. 287-297, 2010.

[38] L. M. Jampol, A. R. Glassman, D. Liu et al., "Diabetic retinopathy clinical Research Network. Plasma vascular endothelial growth factor concentrations after intravitreous anti-vascular endothelial growth factor therapy for diabetic macular edema," Ophthalmology, vol. 125, no. 7, pp. 1054-1063, 2018.

[39] M. I. van der Reis, E. C. La Heij, Y. De Jong-Hesse, P. J. Ringens, F. Hendrikse, and J. S. A. G. Schouten, "A systematic review of the adverse events of intravitreal antivascular endothelial growth factor injections," Retina, vol. 31, no. 8, pp. 1449-1469, 2011.

[40] D. Y. Choi, M. C. Ortube, C. A. McCannel et al., "Sustained elevated intraocular pressures after intravitreal injection of bevacizumab, ranibizumab, and pegaptanib," Retina, vol. 31, no. 6, pp. 1028-1035, 2011.

[41] R. Nuzzi and F. Tridico, "Local and systemic complications after intravitreal administration of anti-vascular endothelial growth factor agents in the treatment of different ocular diseases: a five-year retrospective study," Seminars in Ophthalmology, vol. 30, no. 2, pp. 129-135, 2015.

[42] N. Ferrara, L. Damico, N. Shams, H. Lowman, and R. Kim, "Development of ranibizumab, an anti-vascular endothelial growth factor antigen binding fragment, as therapy for neovascular age-related macular degeneration," Retina, vol. 26, no. 8, pp. 859-870, 2006.

[43] Q. D. Nguyen, S. M. Shah, J. S. Heier et al., "Primary end point (six months) results of the ranibizumab for edema of the mAcula in diabetes (READ-2) study," Ophthalmology, vol. 116, no. 11, pp. 2175-2181, 2009.

[44] P. Massin, F. Bandello, J. G. Garweg et al., "Safety and efficacy of ranibizumab in diabetic macular edema (RESOLVE Study): a 12-month, randomized, controlled, doublemasked, multicenter phase II study," Diabetes Care, vol. 33, no. 11, pp. 2399-2405, 2010.

[45] P. Mitchell, F. Bandello, U. Schmidt-Erfurth et al., "The RESTORE study," Ophthalmology, vol. 118, no. 4, pp. 615-625, 2011.

[46] Q. D. Nguyen, D. M. Brown, D. M. Marcus et al., "Ranibizumab for diabetic macular edema: results from 2 phase III randomized trials: RISE and RIDE," Ophthalmology, vol. 119, no. 4, pp. 789-801, 2012.

[47] Diabetic Retinopathy Clinical Research Network, M. J. Elman, H. Qin, and L. P. Aiello, "Intravitreal ranibizumab for diabetic macular edema with prompt versus deferred laser treatment: three-year randomized trial results," Ophthalmology, vol. 119, no. 11, pp. 2312-2318, 2012.

[48] D. V. Do, Q. D. Nguyen, A. A. Khwaja et al., "Ranibizumab for edema of the macula in diabetes study," JAMA Ophthalmology, vol. 131, no. 2, pp. 139-145, 2013.

[49] D. M. Brown, Q. D. Nguyen, D. M. Marcus et al., "Long-term outcomes of ranibizumab therapy for diabetic macular 
edema: the 36-month results from two phase III trials," Ophthalmology, vol. 120, no. 10, pp. 2013-2022, 2013.

[50] U. Schmidt-Erfurth, G. E. Lang, F. G. Holz et al., "Three-year outcomes of individualized ranibizumab treatment in patients with diabetic macular edema," Ophthalmology, vol. 121, no. 5, pp. 1045-1053, 2014.

[51] O. Comyn, S. Sivaprasad, T. Peto et al., "A randomized trial to assess functional and structural effects of ranibizumab versus laser in diabetic macular edema (the LUCIDATE study)," American Journal of Ophthalmology, vol. 157, no. 5, pp. 960-970, 2014.

[52] M. J. Elman, A. Ayala, N. M. Bressler et al., "Intravitreal ranibizumab for diabetic macular edema with prompt versus deferred laser treatment: 5-year randomized trial results," Ophthalmology, vol. 122, no. 2, pp. 375-381, 2015.

[53] T. Ishibashi, X. Li, A. Koh et al., "The REVEAL study: ranibizumab monotherapy or combined with laser versus laser monotherapy in asian patients with diabetic macular edema," Ophthalmology, vol. 122, no. 7, pp. 1402-1415, 2015.

[54] I. Pearce, S. Banerjee, B. J. L. Burton et al., "Ranibizumab $0.5 \mathrm{mg}$ for diabetic macular edema with bimonthly monitoring after a phase of initial treatment," Ophthalmology, vol. 122, no. 9, pp. 1811-1819, 2015.

[55] C. Prünte, F. Fajnkuchen, S. Mahmood et al., "Ranibizumab $0.5 \mathrm{mg}$ treat-and-extend regimen for diabetic macular oedema: the RETAIN study," British Journal of Ophthalmology, vol. 100, no. 6, pp. 787-795, 2016.

[56] D. V. Do, Y. J. Sepah, Y. J. Sepah et al., "Month-6 primary outcomes of the READ-3 study (Ranibizumab for Edema of the mAcula in Diabetes-Protocol 3 with high dose)," Eye, vol. 29, no. 12, pp. 1538-1544, 2015.

[57] D. S. Boyer, Q. D. Nguyen, D. M. Brown, K. Basu, and J. S. Ehrlich, "Outcomes with as-needed ranibizumab after initial monthly therapy," Ophthalmology, vol. 122, no. 12, pp. 2504-2513, 2015.

[58] H. E. Wiley, D. J. Thompson, C. Bailey et al., "A crossover design for comparative efficacy: a 36-week randomized trial of bevacizumab and ranibizumab for diabetic macular edema," Ophthalmology, vol. 123, no. 4, pp. 841-849, 2016.

[59] V. H. Gonzalez, J. Campbell, N. M. Holekamp et al., "Early and long-term responses to anti-vascular endothelial growth factor therapy in diabetic macular edema: analysis of protocol I data," American Journal of Ophthalmology, vol. 172, pp. 72-79, 2016.

[60] M. Lafuente, L. Ortín, M. Argente et al., "Combined intravitreal ranibizumab and oral supplementation with docosahexaenoic acid and antioxidants for diabetic macular edema," Retina, vol. 37, no. 7, pp. 1277-1286, 2017.

[61] J. F. Payne, C. C. Wykoff, W. L. Clark, B. B. Bruce, D. S. Boyer, and D. M. Brown, "Randomized trial of treat and extend ranibizumab with and without navigated laser for diabetic macular edema," Ophthalmology, vol. 124, no. 1, pp. 74-81, 2017.

[62] C. Fechter, H. Frazier, W. B. Marcus, A. Farooq, H. Singh, and D. M. Marcus, "Ranibizumab $0.3 \mathrm{mg}$ for persistent diabetic macular edema after recent, frequent, and chronic bevacizumab: the ROTATE trial," Ophthalmic Surgery, Lasers and Imaging Retina, vol. 47, no. 11, pp. 1-18, 2016.

[63] G. E. Lang, S. Liakopoulos, J. Vögeler et al., "The RELATION study: efficacy and safety of ranibizumab combined with laser photocoagulation treatment versus laser monotherapy in NPDR and PDR patients with diabetic macular oedema," Acta Ophthalmologica, vol. 96, no. 3, pp. e377-e385, 2018.
[64] X. Li, H. Dai, H. Dai et al., "Efficacy and safety of ranibizumab $0.5 \mathrm{mg}$ in Chinese patients with visual impairment due to diabetic macular edema: results from the 12-month REFINE study," Graefe's Archive for Clinical and Experimental Ophthalmology, vol. 257, no. 3, pp. 529-541, 2019.

[65] S. B. Bressler, T. Almukhtar, A. Bhorade et al., "Repeated intravitreous ranibizumab injections for diabetic macular edema and the risk of sustained elevation of intraocular pressure or the need for ocular hypotensive treatment," JAMA Ophthalmology, vol. 133, no. 5, pp. 589-597, 2015.

[66] H. Guzel, B. Bakbak, M. T. Koylu, S. Gonul, B. Ozturk, and S. Gedik, "The effect and safety of intravitreal injection of ranibizumab and bevacizumab on the corneal endothelium in the treatment of diabetic macular edema," Cutaneous and Ocular Toxicology, vol. 36, no. 1, pp. 5-8, 2017.

[67] R. L. Avery, A. A. Castellarin, N. C. Steinle et al., "Systemic pharmacokinetics following intravitreal injections of ranibizumab, bevacizumab or aflibercept in patients with neovascular AMD," British Journal of Ophthalmology, vol. 98, no. 12, pp. 1636-1641, 2014.

[68] A. N. Economides, L. R. Carpenter, J. S. Rudge et al., "Cytokine traps: multi-component, high-affinity blockers of cytokine action," Nature Medicine, vol. 9, no. 1, pp. 47-52, 2003.

[69] N. Papadopoulos, J. Martin, Q. Ruan et al., "Binding and neutralization of vascular endothelial growth factor (VEGF) and related ligands by VEGF Trap, ranibizumab and bevacizumab," Angiogenesis, vol. 15, no. 2, pp. 171-185, 2012.

[70] J.-F. Korobelnik, D. V. Do, U. Schmidt-Erfurth et al., "Intravitreal aflibercept for diabetic macular edema," Ophthalmology, vol. 121, no. 11, pp. 2247-2254, 2014.

[71] D. M. Brown, U. Schmidt-Erfurth, D. V. Do et al., "Intravitreal aflibercept for diabetic macular edema," Ophthalmology, vol. 122, no. 10, pp. 2044-2052, 2015.

[72] D. V. Do, Q. D. Nguyen, R. Vitti et al., "Intravitreal aflibercept injection in diabetic macular edema patients with and without prior anti-vascular endothelial growth factor treatment," Ophthalmology, vol. 123, no. 4, pp. 850-857, 2016.

[73] C. C. Wykoff, R. T. Le, R. N. Khurana et al., "Outcomes with as-needed aflibercept and macular laser following the phase III VISTA DME trial: ENDURANCE 12-month extension study," American Journal of Ophthalmology, vol. 173, pp. 56-63, 2017.

[74] J. S. Heier, J.-F. Korobelnik, D. M. Brown et al., "Intravitreal aflibercept for diabetic macular edema," Ophthalmology, vol. 123, no. 11, pp. 2376-2385, 2016.

[75] L. M. Jampol, A. R. Glassman, N. M. Bressler, J. A. Wells, and A. R. Ayala, "Anti-vascular endothelial growth factor comparative effectiveness trial for diabetic macular edema," JAMA Ophthalmology, vol. 134, no. 12, p. 1429, 2016.

[76] S. B. Bressler, D. Liu, A. R. Glassman et al., "Change in diabetic retinopathy through 2 years," JAMA Ophthalmology, vol. 135, no. 6, pp. 558-568, 2017.

[77] C. C. Wykoff, W. C. Ou, R. N. Khurana, D. M. Brown, W. Lloyd Clark, and D. S. Boyer, "Long-term outcomes with as-needed aflibercept in diabetic macular oedema: 2-year outcomes of the ENDURANCE extension study," British Journal of Ophthalmology, vol. 102, no. 5, pp. 631-636, 2018.

[78] N. M. Bressler, W. T. Beaulieu, A. R. Glassman et al., "Persistent macular thickening following intravitreous aflibercept, bevacizumab, or ranibizumab for central-involved diabetic macular edema with vision impairment," JAMA Ophthalmology, vol. 136, no. 3, pp. 257-269, 2018. 
[79] R. P. Singh, F. Q. Silva, A. Gibson et al., "Difference in treatment effect between intravitreal aflibercept injection and laser by baseline factors in diabetic macular edema," Ophthalmic Surgery, Lasers and Imaging Retina, vol. 50, no. 3, pp. 167-173, 2019.

[80] C. W. Baker, A. R. Glassman, W. T. Beaulieu et al., "Effect of initial management with aflibercept vs laser photocoagulation vs observation on vision loss among patients with diabetic macular edema involving the center of the macula and good visual acuity," JAMA, vol. 321, no. 19, pp. 1880-1894, 2019.

[81] J.-F. Korobelnik, V. Daien, C. Faure et al., "Real-world outcomes following 12 months of intravitreal aflibercept monotherapy in patients with diabetic macular edema in France: results from the APOLLON study," Graefe's Archive for Clinical and Experimental Ophthalmology, vol. 258, no. 3, p. 521, 2020.

[82] A. M. Mansour, S. I. Al-Ghadban, M. H. Yunis, and M. E. ElSabban, "Ziv-aflibercept in macular disease," British Journal of Ophthalmology, vol. 99, no. 8, pp. 1055-1059, 2015.

[83] A. M. Mansour, M. W. Stewart, M. E. Farah, H. A. Mansour, and J. Chhablani, "Ziv-aflibercept: a cost-effective, off-label, highly potent antagonist of vascular endothelial growth factor," Acta Ophthalmologica, vol. 98, no. 5, 2019.

[84] A. M. Mansour, C. Dedhia, and J. Chhablani, "Three-month outcome of intravitreal ziv-aflibercept in eyes with diabetic macular oedema," British Journal of Ophthalmology, vol. 101, no. 2, pp. 166-169, 2017.

[85] S. R. Singh, G. Chattannavar, A. Ayachit et al., "Intravitreal ziv-aflibercept: safety analysis in eyes receiving more than ten intravitreal injections," Seminars in Ophthalmology, vol. 35, no. 1, pp. 2-6, 2020.

[86] M. Zhang, J. Zhang, M. Yan et al., "A phase 1 study of KH902, a vascular endothelial growth factor receptor decoy, for exudative age-related macular degeneration," Ophthalmology, vol. 118, no. 4, pp. 672-678, 2011.

[87] M. Zhang, D. Yu, C. Yang et al., "The pharmacology study of a new recombinant human VEGF receptor-fc fusion protein on experimental choroidal neovascularization," Pharmaceutical Research, vol. 26, no. 1, pp. 204-210, 2009.

[88] Y. Xu, A. Rong, Y. Bi, and W. Xu, "Intravitreal conbercept injection with and without grid laser photocoagulation in the treatment of diffuse diabetic macular edema in real-life clinical practice," Ophthalmology, vol. 2016, 2016.

[89] Y. Xu, A. Rong, W. Xu, Y. Niu, and Z. Wang, "Comparison of 12-month therapeutic effect of conbercept and ranibizumab for diabetic macular edema: a real-life clinical practice study," BMC Ophthalmology, vol. 17, no. 1, p. 158, 2017.

[90] Q. Zhou, C. Guo, A. You, D Wang, W Wang, and X Zhang, "One-year outcomes of novel VEGF decoy receptor therapy with intravitreal conbercept in diabetic retinopathy-induced macular edema," Molecular Vision, vol. 25, pp. 636-644, 2019.

[91] W.-S. Liu and Y. J. Li, "Comparison of conbercept and ranibizumab for the treatment efficacy of diabetic macular edema: a Meta-analysis and systematic review," International Journal of Ophthalmology, vol. 12, no. 9, pp. 1479-1486, 2019.

[92] F. Li, L. Zhang, Y. Wang et al., "One-year outcome of conbercept therapy for diabetic macular edema," Current Eye Research, vol. 43, no. 2, pp. 218-223, 2018.

[93] X. Sun, J. Zhang, J. Tian, S. Chen, F. Zeng, and G. Yuan, "Comparison of the efficacy and safety of intravitreal conbercept with intravitreal ranibizumab for treatment of diabetic macular edema: a meta-analysis," Journal of Ophthalmology, vol. 2020, 2020.

[94] H. J. Sohn, D. H. Han, I. T. Kim et al., "Changes in aqueous concentrations of various cytokines after intravitreal triamcinolone versus bevacizumab for diabetic macular edema," American Journal of Ophthalmology, vol. 152, no. 4, pp. 686-694, 2011.

[95] H. L. Brooks Jr., S. Caballero Jr., C. K. Newell et al., "Vitreous levels of vascular endothelial growth factor and stromalDerivedFactor 1 in patients with diabetic retinopathy and cystoid macular edema beforeand after intraocular injection of triamcinolone," Archives of Ophthalmology, vol. 122, no. 12, pp. 1801-1807, 2004.

[96] M. Nauck, G. Karakiulakis, A. P. Perruchoud, E. Papakonstantinou, and M. Roth, "Corticosteroids inhibit the expression of the vascular endothelial growth factor gene in human vascular smooth muscle cells," Archives of Ophthalmology, vol. 341, no. 2-3, pp. 309-315, 1998.

[97] D. Dyer, D. Callanan, T. Bochow et al., "Clinical evaluation of the safety and efficacy of preservative-free triamcinolone (triesence [triamcinolone acetonide injectable suspension] $40 \mathrm{Mg} / \mathrm{ml}$ ) for visualization during pars plana vitrectomy," Retina, vol. 29, no. 1, pp. 38-45, 2009.

[98] N. Haghjou, M. Soheilian, and M. J. Abdekhodaie, "Sustained release intraocular drug delivery devices for treatment of uveitis," Journal of Ophthalmic \& Vision Research, vol. 6, no. 4, pp. 317-329, 2011.

[99] J.-E. Chang-Lin, M. Attar, A. A. Acheampong et al., "Pharmacokinetics and pharmacodynamics of a sustainedrelease dexamethasone intravitreal implant," Investigative Opthalmology \& Visual Science, vol. 52, no. 1, pp. 80-86, 2011.

[100] D. S. Boyer, Y. H. Yoon, R. Belfort Jr. et al., "Three-year, randomized, sham-controlled trial of dexamethasone intravitreal implant in patients with diabetic macular edema," Ophthalmology, vol. 121, no. 10, pp. 1904-1914, 2014.

[101] J. A. Haller, B. D. Kuppermann, M. S. Blumenkranz et al., "Randomized controlled trial of an intravitreous dexamethasone drug delivery system in patients with diabetic macular edema," Archives of Ophthalmology, vol. 128, no. 3, pp. 289-296, 2010.

[102] D. S. Boyer, D. Faber, S. Gupta et al., "Dexamethasone intravitreal implant for treatment of diabetic macular edema in vitrectomized patients," Retina, vol. 31, no. 5, pp. 915-923, 2011.

[103] D. G. Callanan, S. Gupta, D. S. Boyer et al., "Dexamethasone intravitreal implant in combination with laser photocoagulation for the treatment of diffuse diabetic macular edema," Ophthalmology, vol. 120, no. 9, pp. 1843-1851, 2013.

[104] M. C. Gillies, L. L. Lim, A. Campain et al., “A randomized clinical trial of intravitreal bevacizumab versus intravitreal dexamethasone for diabetic macular edema," Ophthalmology, vol. 121, no. 12, pp. 2473-2481, 2014.

[105] S. Fraser-Bell, L. L. Lim, A. Campain et al., "Bevacizumab or dexamethasone implants for DME: 2-year results (the BEVORDEX study)," Ophthalmology, vol. 123, no. 6, pp. 1399-1401, 2016.

[106] R. Lazic, M. Lukic, I. Boras et al., "Treatment of anti-vascular endothelial growth factor-resistant diabetic macular edema with dexamethasone intravitreal implant," Retina, vol. 34, no. 4, pp. 719-724, 2014.

[107] S. U. Shah, A. Harless, L. Bleau, and R. K. Maturi, "Prospective randomized subject-masked study OF intravitreal 
bevacizumab monotherapy versus dexamethasone implant monotherapy IN the treatment OF persistent diabetic macular edema," Retina, vol. 36, no. 10, pp. 1986-1996, 2016.

[108] R. K. Maturi, L. Bleau, J. Saunders, M. Mubasher, and M. W. Stewart, "A 12-MONTH, single-masked, randomized controlled study OF eyes with persistent diabetic macular edema after multiple anti-vegf injections to assess the efficacy OF the dexamethasone-delayed delivery system AS an adjunct to bevacizumab compared with continued bevacizumab monotherapy," Retina, vol. 35, no. 8, pp. 1604-1614, 2015.

[109] R. P. Danis, S. Sadda, X.-Y. Li, H. Cui, Y. Hashad, and S. M. Whitcup, "Anatomical effects of dexamethasone intravitreal implant in diabetic macular oedema: a pooled analysis of 3-year phase III trials," British Journal of Ophthalmology, vol. 100, no. 6, pp. 796-801, 2016.

[110] V. Sarao, D. Veritti, C. Furino et al., "Dexamethasone implant with fixed or individualized regimen in the treatment of diabetic macular oedema: six-month outcomes of the UDBASA study," Acta Ophthalmologica, vol. 95, no. 4, pp. e255-e260, 2017.

[111] R. K. Maturi, A. R. Glassman, D. Liu et al., "Effect of adding dexamethasone to continued ranibizumab treatment in patients with persistent diabetic macular edema," JAMA Ophthalmology, vol. 136, no. 1, pp. 29-38, 2018.

[112] P. A. Campochiaro, D. M. Brown, A. Pearson et al., "Longterm benefit of sustained-delivery fluocinolone acetonide vitreous inserts for diabetic macular edema," Ophthalmology, vol. 118, no. 4, pp. 626-635, 2011.

[113] J. J. Escobar-Barranco, B. Pina-Marín, and M. FernándezBonet, "Dexamethasone implants in patients with naïve or refractory diffuse diabetic macular edema," Ophthalmologica, vol. 233, no. 3-4, pp. 176-185, 2015.

[114] S. Guigou, S. Pommier, F. Meyer et al., "Efficacy and safety of intravitreal dexamethasone implant in patients with diabetic macular edema," Ophthalmologica, vol. 233, no. 3-4, pp. 169-175, 2015.

[115] J. Chhablani, P. Bansal, D. Veritti et al., "Dexamethasone implant in diabetic macular edema in real-life situations," Eye, vol. 30, no. 3, pp. 426-430, 2016.

[116] A. Malclès, C. Dot, N. Voirin et al., "Real-life study in diabetic macular edema treated with dexamethasone implant," Retina, vol. 37, no. 4, pp. 753-760, 2017.

[117] A. Pareja-Ríos, P. Ruiz-de la Fuente-Rodríguez, S. BonaqueGonzález et al., "Intravitreal dexamethasone implants for diabetic macular edema," Archives of Ophthalmology, vol. 11, no. 1, pp. 77-82, 2018.

[118] M. Iglicki, C. Busch, D. Zur et al., "Dexamethasone implant for diabetic macular edema in naive compared with refractory eyes," Retina, vol. 39, no. 1, pp. 44-51, 2019.

[119] M. Nagpal, N. Mehrotra, R. Juneja, and H. Jain, "Dexamethasone implant $(0.7 \mathrm{mg})$ in Indian patients with macular edema: real-life scenario," Taiwan Journal of Ophthalmology, vol. 8, no. 3, pp. 141-148, 2018.

[120] V. Castro-Navarro, E. Cervera-Taulet, C. Navarro-Palop, C. Monferrer-Adsuara, L. Hernández-Bel, and J. MonteroHernández, "Intravitreal dexamethasone implant Ozurdex in naïve and refractory patients with different subtypes of diabetic macular edema," BMC Ophthalmology, vol. 19, no. 1, p. 15, 2019.

[121] C. Busch, D. Zur, D. Zur et al., "Shall we stay, or shall we switch? Continued anti-VEGF therapy versus early switch to dexamethasone implant in refractory diabetic macular edema," Acta Diabetologica, vol. 55, no. 8, pp. 789-796, 2018.

[122] A. Hernández Martínez, E. Pereira Delgado, G. Silva Silva et al., "Early versus late switch: how long should we extend the anti-vascular endothelial growth factor therapy in unresponsive diabetic macular edema patients?" European Journal of Ophthalmology, vol. 30, no. 5, 2019.

[123] G. Demir, A. Ozkaya, E. Yuksel et al., "Early and late switch from ranibizumab to an intravitreal dexamethasone implant in patients with diabetic macular edema in the event of a poor anatomical response," Clinical Drug Investigation, vol. 40, no. 2, pp. 119-128, 2020.

[124] J. Ruiz-Medrano, R. Rodríguez-Leor, E. Almazán et al., "Results of dexamethasone intravitreal implant (Ozurdex) in diabetic macular edema patients: early versus late switch," European Journal of Ophthalmology, vol. 2020, 2020.

[125] S. R. Peterson, P. A. Silva, T. J. Murtha, and J. K. Sun, "Cataract surgery in patients with diabetes: management strategies," Seminars in Ophthalmology, vol. 33, no. 1, pp. 75-82, 2018.

[126] A. K. Denniston, A. Y. Lee, C. S. Lee et al., "United Kingdom Diabetic Retinopathy Electronic Medical Record (UK DR EMR) Users Group: report 4, real-world data on the impact of deprivation on the presentation of diabetic eye disease at hospital services," British Journal of Ophthalmology, vol. 103, no. 6, pp. 837-843, 2019.

[127] H. Kiziltoprak, K. Tekin, M. Inanc, and Y. S. Goker, "Cataract in diabetes mellitus," World Journal of Diabetes, vol. 10, no. 3, pp. 140-153, 2019.

[128] C. W. Baker, T. Almukhtar, N. M. Bressler et al., "Macular edema after cataract surgery in eyes without preoperative central-involved diabetic macular edema," JAMA Ophthalmology, vol. 131, no. 7, pp. 870-879, 2013.

[129] G. A. Panozzo, E. Gusson, G. Panozzo, and G. Dalla Mura, "Dexamethasone intravitreal implant at the time of cataract surgery in eyes with diabetic macular edema," European Journal of Ophthalmology, vol. 27, no. 4, pp. 433-437, 2017.

[130] C. Furino, F. Boscia, A. Niro et al., "Combined phacoemulsification and intravitreal dexamethasone implant (Ozurdex) in diabetic patients with coexisting cataract and diabetic macular edema," Journal of Ophthalmology, vol. 2017, p. 1, 2017.

[131] P. Calvo, A. Ferreras, F. Al Adel, W. Dangboon, and M. H. Brent, "Effect OF an intravitreal dexamethasone implant ON diabetic macular edema after cataract surgery," Retina, vol. 38, no. 3, pp. 490-496, 2018.

[132] C. Furino, F. Boscia, A. Niro et al., "Diabetic macular edema and cataract surgery: phacoemulsification combined with dexamethasone intravitreal implant compared with standard phacoemulsification," Retina, vol. 2020, 2020.

[133] J. Cunha-Vaz, P. Ashton, R. Iezzi et al., "Sustained delivery fluocinolone acetonide vitreous implants," Ophthalmology, vol. 121, no. 10, pp. 1892-1903, 2014.

[134] Y. Yang, C. Bailey, C. Bailey et al., "Long-term outcomes of phakic patients with diabetic macular oedema treated with intravitreal fluocinolone acetonide (FAc) implants," Eye, vol. 29, no. 9, pp. 1173-1180, 2015.

[135] R. K. Parrish 2nd., P. A. Campochiaro, P. A. Pearson, K. Green, and C. E. Traverso, "Characterization of intraocular pressure increases and management strategies following treatment with fluocinolone acetonide intravitreal implants in the FAME trials," Ophthalmic Surgery, Lasers and Imaging Retina, vol. 47, no. 5, pp. 426-435, 2016.

[136] P. A. Pearson, T. L. Comstock, M. Ip et al., "Fluocinolone acetonide intravitreal implant for diabetic macular edema: a 3-year multicenter, randomized, controlled clinical trial," Ophthalmology, vol. 118, no. 8, pp. 1580-1587, 2011. 
[137] Iluvien (fluocinolone acetonide intravitreal implant) $0.19 \mathrm{mg}$ for intravitreal injection data sheet, 2020, https://webcache. googleusercontent.com/search?q=cache:gu3-KoUuX6gJ:https:// www.alimerasciences.com/wp-content/uploads/2016/02/Prescri bing-Information.pdf $+\& \mathrm{~cd}=1 \& \mathrm{hl}=\mathrm{es} \& \mathrm{ct}=\mathrm{clnk} \& \mathrm{gl}=\mathrm{es}$.

[138] P. A. Campochiaro, G. Hafiz, S. M. Shah et al., "Sustained ocular delivery of fluocinolone acetonide by an intravitreal insert," Ophthalmology, vol. 117, no. 7, pp. 1393-1399, 2010.

[139] W. Fusi-Rubiano, C. Mukherjee, M. Lane et al., "Treating Diabetic Macular Oedema (DMO): real world UK clinical outcomes for the $0.19 \mathrm{mg}$ Fluocinolone Acetonide intravitreal implant (Iluvien) at 2 years," BMC Ophthalmology, vol. 18, no. 1 , p. $62,2018$.

[140] M. S. Habib, "ILUVIEN technology in the treatment of center-involving diabetic macular edema: a review of the literature," Therapeutic Delivery, vol. 9, no. 8, pp. 547-556, 2018.

[141] W. Fusi-Rubiano, R. R. Blow, M. Lane, R. Morjaria, and A. K. Denniston, "Iluvien (fluocinolone acetonide $0.19 \mathrm{mg}$ intravitreal implant) in the treatment of diabetic macular edema: a review," Ophthalmology and Therapy, vol. 7, no. 2, pp. 293-305, 2018.

[142] H. Massa, A. M. Nagar, A. Vergados, P. Dadoukis, S. Patra, and G. D. Panos, "Intravitreal fluocinolone acetonide implant (ILUVIEN) for diabetic macular oedema: a literature review," Journal of International Medical Research, vol. 47, no. 1, pp. 31-43, 2019.

[143] J. F. Young, A. Walkden, A. Stone, and S. Mahmood, "Clinical effectiveness of intravitreal fluocinolone acetonide (FAc) (ILUVIEN) in patients with diabetic macular oedema (DMO) refractory to prior therapy: the manchester experience," Ophthalmology and Therapy, vol. 8, no. 3, pp. 477-484, 2019.

[144] A. J. Augustin, S. Bopp, M. Fechner et al., "Three-year results from the Retro-IDEAL study: real-world data from diabetic macular edema (DME) patients treated with ILUVIEN (0.19 mg fluocinolone acetonide implant)," European Journal of Ophthalmology, vol. 30, no. 2, 2019.

[145] S. E. Holden, B. Kapik, A. B. Beiderbeck, and C. J. Currie, "Comparison of data characterizing the clinical effectiveness of the fluocinolone intravitreal implant (ILUVIEN) in patients with diabetic macular edema from the real world, noninterventional ICE-UK study and the FAME randomized controlled trials," Current Medical Research and Opinion, vol. 35, no. 7, pp. 1165-1176, 2019.

[146] U. Chakravarthy, S. R. Taylor, F. H. J. Koch, J. P. Castro de Sousa, and C. Bailey, "Changes in intraocular pressure after intravitreal fluocinolone acetonide (ILUVIEN): real-world experience in three European countries," British Journal of Ophthalmology, vol. 103, no. 8, pp. 1072-1077, 2019.

[147] J. Coelho, L. Malheiro, J. Melo Beirão, A. Meireles, and B. Pessoa, "Real-world retrospective comparison of $0.19 \mathrm{mg}$ fluocinolone acetonide and $0.7 \mathrm{mg}$ dexamethasone intravitreal implants for the treatment of diabetic macular edema in vitrectomized eyes," Clinical Ophthalmology, vol. 13, pp. 1751-1759, 2019.

[148] G. D. Panos, N. Arruti, and S. Patra, "The long-term efficacy and safety of fluocinolone acetonide intravitreal implant 190 $\mu \mathrm{g}$ (ILUVIEN) in diabetic macular oedema in a multi-ethnic inner-city population," European Journal of Ophthalmology, vol. 2020, 2020.

[149] H. K. Binz, M. T. Stumpp, P. Forrer, P. Amstutz, and A. Plückthun, "Designing repeat proteins: well-expressed, soluble and stable proteins from combinatorial libraries of consensus ankyrin repeat proteins," Journal of Molecular Biology, vol. 332, no. 2, pp. 489-503, 2003.

[150] M. T. Stumpp, H. K. Binz, and P. Amstutz, "DARPins: a new generation of protein therapeutics," Drug Discover Today, vol. 13, no. 15-16, pp. 695-701, 2008.

[151] J. A. Hammill, H. VanSeggelen, C. W. Helsen et al., "Designed ankyrin repeat proteins are effective targeting elements for chimeric antigen receptors," Journal of Immunother Cancer, vol. 3, p. 55, 2015.

[152] R. Sacconi, C. Giuffrè, E. Corbelli, E. Borrelli, G. Querques, and F. Bandello, "Emerging therapies in the management of macular edema: a review," F1000Research, vol. 8, p. 1413, 2019.

[153] P. A. Campochiaro, R. Channa, B. B. Berger et al., "Treatment of diabetic macular edema with a designed ankyrin repeat protein that binds vascular endothelial growth factor: a phase I/II study," American Journal of Ophthalmology, vol. 155, no. 4, pp. 697-704, 2013.

[154] T. S. Hassan, "A multicenter, double masked phase 2 clinical trial evaluating abicipar pegol for diabetic macular edema," 2020.

[155] A. Markham, "Brolucizumab: first approval," Drugs, vol. 79, no. 18, pp. 1997-2000, 2019.

[156] P. U. Dugel, G. J. Jaffe, P. Sallstig et al., "Brolucizumab versus aflibercept in participants with neovascular age-related macular degeneration: a randomized trial," Ophthalmology, vol. 124, no. 9, pp. 1296-1304, 2017.

[157] P. U. Dugel, A. Koh, Y. Ogura et al., "HAWK and HARRIER: phase 3, multicenter, randomized, double-masked trials of brolucizumab for neovascular age-related macular degeneration," Ophthalmology, vol. 127, no. 1, pp. 72-84, 2020.

[158] A. J. Witkin, P. Hahn, T. G. Murray et al., "Occlusive retinal vasculitis following intravitreal brolucizumab," Journal of VitreoRetinal Diseases, vol. 4, no. 4, pp. 269-279, 2020.

[159] C. R. Baumal, R. F. Spaide, L. Vajzovic et al., "Retinal vasculitis and intraocular inflammation after intravitreal injection of brolucizumab," Ophthalmology, vol. 127, no. 10, pp. 1345-1359, 2020.

[160] Novartis Pharmaceuticals, "Study of efficacy and safety of Brolucizumab vs. Aflibercept in Patients with visual impairment due to diabetic macular edema (KESTREL)," 2020.

[161] J. G. Garweg, "A randomized, double-masked, multicenter, phase III study assessing the efficacy and safety of brolucizumab versus aflibercept in patients with visual impairment due to diabetic macular edema (KITE)," Klinische Monatsblätter für Augenheilkunde, vol. 237, no. 4, pp. 450-453, 2020.

[162] S. Rangasamy, R. Srinivasan, J. Maestas, P. G. McGuire, and A. Das, "A potential role for angiopoietin 2 in the regulation of the blood-retinal barrier in diabetic retinopathy," Investigative Opthalmology \& Visual Science, vol. 52, no. 6, pp. 3784-3791, 2011.

[163] J. Sahni, S. S. Patel, P. U. Dugel et al., "Simultaneous inhibition of angiopoietin-2 and vascular endothelial growth factor-A with faricimab in diabetic macular edema," Ophthalmology, vol. 126, no. 8, pp. 1155-1170, 2019. 